TAUP 2926- 10

\title{
Can holography reproduce the QCD Wilson line?
}

\author{
Uri Kol and Jacob Sonnenschein \\ School of Physics and Astronomy, \\ The Raymond and Beverly Sackler Faculty of Exact Sciences, \\ Tel Aviv University, Ramat Aviv 69978, Israel \\ E-mail: urikol@post.tau.ac.il, cobi@post.tau.ac.il
}

\begin{abstract}
Recently a remarkable agreement was found between lattice simulations of long Wilson lines and behavior of the Nambu Goto string in flat space-time. However, the latter fails to fit the short distance behavior since it admits a tachyonic mode for a string shorter than a critical length. In this paper we examine the question of whether a classical holographic Wilson line can reproduce the lattice results for Wilson lines of any length. We determine the condition on the the gravitational background to admit a Coulombic potential at short distances. We analyze the system using three different renormalization schemes. We perform an explicit best fit comparison of the lattice results with the holographic models based on near extremal $D_{3}$ and $D_{4}$ branes, non-critical near extremal $A d S_{6}$ model and the Klebanov Strassler model. We find that all the holographic models examined admit after renormalization a constant term in the potential. We argue that the curves of the lattice simulation also have such a constant term and we discuss its physical interpretation.
\end{abstract}




\section{Contents}

1 Introduction 1

2 The holographic Wilson line 3

3 The conditions on the background to satisfy a coulombic potential $\begin{array}{ll}\text { at small distances } & 7\end{array}$

4 Renormalization $\quad 8$

4.1 Holographic renormaliztion $\quad 9$

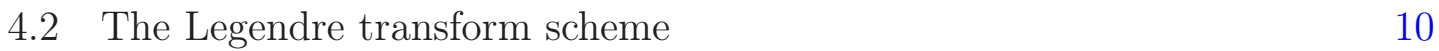

$\begin{array}{ll}4.3 \text { The mass subtraction scheme } & 11\end{array}$

$\begin{array}{lll}4.4 \text { Conclusions } & 12\end{array}$

5 The Wilson line of the holographic models 13

6 Units of measurement 17

7 Comparison with lattice gauge theories $\quad 20$

8 The constant terms $\quad 25$

9 Concluding remarks \& future directions 28

A The conditions on the background to satisfy a coulombic potential $\begin{array}{ll}\text { in the UV } & 31\end{array}$

B Conical singularity in near extremal $D_{p}$ brane 32

C Analytical expressions for energy and length of the string in near extremal $D_{p}$ brane $\quad 33$

D The values of the constant terms in near extremal $D_{p}$ brane $\quad 35$

E Analytical expressions for energy and length of the string in noncritical $A d S_{n+1} \quad 36$

F The values of the constant terms in non-critical $A d S_{n+1} \quad 37$

G The Wilson line of the Klebanov Strassler background 38 


\section{Introduction}

Recently lattice simulations of Wilson lines were found to admit a remarkable agreement with the relation between the energy and the length of the string as follows from the Nambu Goto (N.G.) formulation. The simulations measured this relation in two [1] and three [2] space dimensions for both closed strings wounded along a circle as well as for the open string case [3]. The dependence of the energy on the distance between the endpoints of an open bosonic quantum string in $d$ dimensional flat space-time is given by [4]

$$
E=\sqrt{(\sigma \ell)^{2}+\left(n-\frac{d-2}{24}\right) 8 \pi \sigma}
$$

where $\sigma$ is the string tension, $\ell$ is the separation distance and $n$ is the excitation level. It was found out that for the ground state the agreement with the full squareroot formula is better than the agreement with any approximation of this formula for long strings $\left(\sigma \ell^{2}>>1\right)$, like $E \sim \sigma \ell+\frac{(d-2)}{24} \frac{4 \pi}{\ell}+\frac{(d-2)}{24} \frac{8 \pi^{2}}{\sigma \ell^{3}}+\ldots$. What is in particular remarkable is the fact that the agreement holds also for short distances, up to $\ell \sqrt{\sigma} \sim 1.6$.

Note however that the N.G. behavior (1.1) cannot serve as an adequate description for distances shorter than the critical length

$$
\ell_{c r}=\frac{\pi(d-2)}{3} \frac{1}{\sqrt{\sigma}}
$$

since there the energy develops an imaginary part. It is thus clear that to accommodate the short range behavior of the potential one needs to go beyond the stringy picture of above. A potential solution to this problem is to replace the string that resides in four dimensional flat space-time with a holographic string in non-flat higher dimensional space-time. An argument in favor of this scenario is the fact that the behavior of the Wilson line in an $A d S_{5}$ space admits a $\frac{1}{\ell}$ which is similar to the behavior of the quark anti-quark potential in short distances, namely $E \sim \frac{\alpha(\ell)}{\ell}$ where $\alpha(\ell)$ is the running coupling. This naturally calls for a holographic description in terms of a background that admits a confining behavior [5] for long strings and which is asymptotically $A d S$.

Precise lattice simulations of the quark anti-quark potential especially in the short distance range may serve as a test of the idea of weakly curved holography. Whereas for long strings the contributions of massive stringy modes are negligible, for short strings the impact of the massive modes becomes more important. In holographic models massive modes arise from fluctuations of the string along the radial direction, compact directions and along fermionic directions. Thus high precision profile for short distances will enable one to rule out or confirm the idea of describing the Wilson line in terms of a string in weakly curved higher dimensional space-time. 
The goal of this paper is to search for a holographic background that admits a Wilson line which is as close as possible to the potential deduced from lattice calculations and which at the same time is in accordance with other requirements. In [5] the conditions for a background to admit an area law behavior for long Wilson lines were stated. For short Wilson lines the holographic model is required to mimic the QCD perturbative behavior of the potential. To determine this condition is a complicated task. Here instead, in order to have a guide in the search of the appropriate background, we determine the conditions for a background for which the short distance potential behaves as $\frac{1}{\ell}$.

In the UV the lattice simulates QCD in its perturbative regime. The holographic correspondence maps this regime to a highly curved gravitational background. Thus obviously the comparison we make to the holographic stringy result is on a solid ground only for large and maybe intermediate distances. One may wonder whether any reliable information can be extracted from the stringy picture in this case. Obviously the background will be determined in this case by an action that includes higher order curvature terms and moreover the stringy calculation will include higher $\alpha^{\prime}$ corrections. However, it is quite plausible that the maximally symmetric $A d S$ background will remain a solution thought the radius will differ from the classical value. For such a case, even though the N.G. is not an adequate framework of calculation, due to scale invariance the energy should scale as a $\frac{1}{\ell}$.

It is well known since the seminal paper [6] that the classical string action that corresponds to a Wilson line diverges, and hence one has to invoke some renormalization procedure. We discuss three different possible renormalization schemes: the holographic renormalization of [7], the Legender transform approach [8] and the mass subtraction [9]. It is shown that for backgrounds without an horizon ${ }^{1}$ the three schemes yield the same result but for cases that have horizon the last prescription differs from the two previous ones. From the large arsenal of holographic models we have analyzed the models of near extremal $D_{3}$ and $D_{4}$ branes [10], non-critical near extremal $A d S_{6}[11,12]$ and the Klebanov Strassler (KS) background [13] ${ }^{2}$.

An outcome of this analysis is the fact that the quark anti-quark potential admits on top of the linear and $\frac{1}{\ell}$ terms also a constant term. This constant term appears in all the holographic backgrounds we have analyzed and in all the renormalization schemes. It also follows from the lattice simulations, where it is defined as the difference between the constants added to find the best fit with the simulation data in the large $\ell$ range and in the short range. However, it seems that the constant term in the latter range is not well-defined in field theory as the theoretical perturbative expansion is not guaranteed to converge. Being aware of this point we suggest dif-

\footnotetext{
${ }^{1}$ Horizon in general relativity associates with the time component of the metric. Here and in the rest of the paper horizon will relate to a space coordinate rather than to the time.

${ }^{2}$ Some similar aspects of the Wilson line in the KS background have already been discussed in [14].
} 
ferent ways to discuss the constant term in spite of this problem. The fact that the constants in the two different ranges are different is what renders this constant to be a physical quantity. A possible physical interpretation of this constant is in terms of masses attached to the string endpoints. A similar constant term has also been treated in the work of [15] on the zero point energy of renormalized Wilson loops, where the discussed action is a flat spacetime N.G. plus higher terms in covariant derivatives on the worldsheet.

Quantitative comparison between the lattice simulations and the holographic results shows that the Klebanov Strassler background exhibits the smallest $\chi$ square. However, the non-critical $A d S_{6}$ model also admits a reasonable fit to the simulation results and it has the advantage of being asymptotically $A d S$, while the $K S$ is only approximately asymptotically $A d S$ and hence doesn't protected from quantum corrections in the $U V$. Both models are also invariant under four dimensional Poincare symmetry, which is an essential requirement.

The paper is organized as follows. After this introduction, in section (2) we review the notion of Wilson loops in holographic models. We then discuss the conditions on the background to satisfy a coulombic potential at small distances. The issue of the holographic renormalization is discussed in section (4). We analyze separately the various renormalization schemes of [7], [8] and [9]. A brief review of certain holographic models dual of Yang Mills like theories and discussion about their Wilson line is presented in section (5). In section (6) prior to the performing of the comparison between the outcome of holography and the results of lattice simulation, we setup the units appropriate for the comparison. The latter is made in section (7). In section (8) we discuss the physical constant that follows from the simulations and the holographic models. We end the paper in section (9) with a brief summary and discussion of the findings of this paper and certain open questions. In particular we discuss the issue of the impact of quantum fluctuations on the holographic Wilson line.

\section{The holographic Wilson line}

We will investigate several holographic models, all of them are of the form of some high dimensional space with a boundary that consists some of the coordinates (from which only four should eventually be infinite), a radial coordiante that takes us from the bulk of the space to the boundary and can be viewed as energy scale and possibly some other coordinates transverse to the boundary's coordinates. Following [5] we will assume that the metric depends only on the radial coordinate such that its general form is

$$
d s^{2}=-G_{t t}(u) d t^{2}+G_{u u}(u) d u^{2}+G_{x_{||} x_{||}}(u) d x_{\|}^{2}+G_{x_{T} x_{T}}(u) d x_{T}^{2}
$$


where $t$ is the time direction, $u$ is the radial coordinate, $x_{\|}$are the coordinates on the boundary and $x_{T}$ are the transverse coordiantes. We adopt the notation in which the radial coordinate is positive defined and the boundary is located at $u=\infty$. In addition, an "horizon" may exist at $u=u_{\Lambda}$, such that spacetime is defined in the region $u_{\Lambda}<u<\infty$ instead of $0<u<\infty$ in the case where no horizon is present. In order to make our discussion most general, we will consider the case with an existing horizon. Later on, when we imply the general analysis to specific models, we would just set $u_{\Lambda}$ to zero in cases with no horizon.

The construction we will examine is of an open string living in the bulk of the space with its both ends tied to the boundary. From the viewpoint of the field theory living on the boundary the endpoints of the string are the $q \bar{q}$ pair, so the energy of the string is related to the energy of the pair [5]. In order to calculate the energy of this configuration in the classical level we will use the notations and results of [5]. First, we define

$$
\begin{aligned}
f^{2}(u) & \equiv G_{t t}(u) G_{x_{||} x_{||}}(u) \\
g^{2}(u) & \equiv G_{t t}(u) G_{u u}(u)
\end{aligned}
$$

Upon choosing the worldsheet coordinates $\sigma=x$ ( $x$ is a coordinate on the boundary pointing in the direction from one endpoint of the string to the other one) and $\tau=t$ and assuming translation invariance along $t$, the Nambu-Goto action describing the string takes the form

$$
\begin{aligned}
S_{N G} & =\int d \sigma d \tau \sqrt{\operatorname{det}\left[\partial_{\alpha} X^{\mu} \partial_{\beta} X^{\nu} G_{\mu \nu}\right]} \\
& =\int d x d t \sqrt{f^{2}(u(x))+g^{2}(u(x))\left(\partial_{x} u\right)^{2}} \\
& =T \int d x \mathcal{L}
\end{aligned}
$$

Then the conjugate momentum and the Hamiltonian are

$$
\begin{gathered}
p=\frac{\delta \mathcal{L}}{\delta u^{\prime}}=\frac{g^{2}(u) u^{\prime}}{\sqrt{f^{2}(u)+g^{2}(u) u^{\prime 2}}} \\
\mathcal{H}=p \cdot u^{\prime}-\mathcal{L}=-\frac{f^{2}(u)}{\mathcal{L}}
\end{gathered}
$$

As the Hamiltonian does not depend explicitly on $x$, its value is a constant of motion. We shall deal with the case in which $u(x)$ is an even function, and therefore there is a minimal value $u_{0}=u(0)$ for which $u^{\prime}(0)=0$. At that point we see from (2.4) that $p=0$. The constant of motion is therefore

$$
\mathcal{H}=-f\left(u_{0}\right)
$$

from which we can extract the differential equation of the geodesic line

$$
\frac{d u}{d x}= \pm \frac{f(u)}{g(u)} \frac{\sqrt{f^{2}(u)-f^{2}\left(u_{0}\right)}}{f\left(u_{0}\right)}
$$


and re-express the on-shell lagrangian (i.e. the lagrangian on the equation of motion) as a function of $f(u)$ only

$$
\mathcal{L}=\frac{f^{2}(u)}{f\left(u_{0}\right)}
$$

Then the distance between the string's endpoints (or the distance between the "quarks") would be

$$
\ell\left(u_{0}\right)=\int d x=\int d u\left(\frac{d u}{d x}\right)^{-1}=2 \frac{1}{f\left(u_{0}\right)} \int_{u_{0}}^{u_{B}} d u \frac{f^{2}\left(u_{0}\right)}{f^{2}(u)} \frac{g(u)}{\sqrt{1-\left(\frac{f\left(u_{0}\right)}{f(u)}\right)^{2}}}
$$

where $u_{0}$ is the minimal value in the radial direction to which the string reaches and $u_{b}$ is the value of $u$ on the boundary. The bare energy of the string is given by

$$
\begin{aligned}
E_{\text {bare }}\left(u_{0}\right) & =\int d x \mathcal{L}=\int d u\left(\frac{d u}{d x}\right)^{-1} \mathcal{L}=2 \int_{u_{0}}^{u_{b}} d u \frac{g(u)}{\sqrt{1-\left(\frac{f\left(u_{0}\right)}{f(u)}\right)^{2}}} \\
& =f\left(u_{0}\right) \cdot \ell\left(u_{0}\right)+2 \int_{u_{0}}^{u_{b}} d u g(u) \sqrt{1-\frac{f^{2}\left(u_{0}\right)}{f^{2}(u)}}
\end{aligned}
$$

Generically, the bare energy diverges and hence a renormalization procedure is needed. Possible renormalization schemes are discussed in section (4). Here we follow [5] and use the mass subtraction scheme in which the bare masses of the quarks are subtracted from the bare energy. The bare quark mass is viewed as a straight string with a constant value of $x$, stretching from $u=0$ (or $u=u_{\Lambda}$ if there exists an horizon at $u_{\Lambda}$ ) to $u=u_{b}$, such that it is given by

$$
m_{q}=\int_{u_{\Lambda}}^{u_{b}} d u g(u)
$$

Then the renormalized energy would be given by

$$
E\left(u_{0}\right)=f\left(u_{0}\right) \cdot \ell\left(u_{0}\right)-2 \mathcal{K}\left(u_{0}\right)
$$

where $\mathcal{K}\left(u_{0}\right)$ is

$$
\mathcal{K}\left(u_{0}\right)=\int_{u_{0}}^{u_{b}} d u g(u)\left(1-\sqrt{1-\frac{f^{2}\left(u_{0}\right)}{f^{2}(u)}}\right)+\int_{u_{\Lambda}}^{u_{0}} d u g(u)
$$

In section (4) we make a discussion about the differences between different renormalization schemes. We emphasize that this result for the energy is only at the classical level and do not include quantum corrections.

In order to reproduce the QCD heavy quarks potential we first have to demand the holographic models to reproduce the asymptotic forms of the potential. This leads to several restrictions on the forms of the $f$ and $g$ functions. The condition for confining behavior at large distances was derived in [5]: 

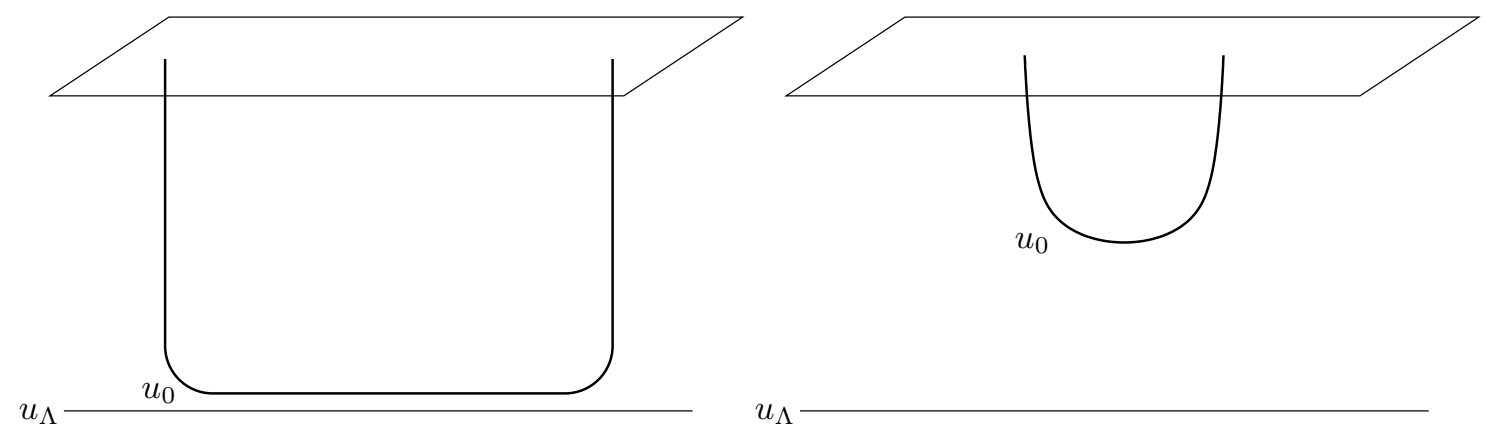

Figure 1. The physical picture arising from the present construction. Left: The confining limit is approached as the string is close to the horizon, then most of the string lies on the horizon and implies a string-like interaction between the quarks (the endpoints of the string). Right: The conformal limit is reconstructed when the string is far away from the horizon such that it is ruled by the geometry near the boundary.

1. $f$ has a minimum at $u_{\text {min }}$ and $f\left(u_{\text {min }}\right) \neq 0$ or

2. $g$ diverges at $u_{\text {div }}$ and $f\left(u_{\text {div }}\right) \neq 0$

Then the string tension is given by $f\left(u_{\text {min }}\right)$ or $f\left(u_{\text {div }}\right)$, correspondingly. The second asymptotic is perturbative QCD at small distances. In section (3) we derive the conditions on the background to reproduce the leading perturbative behavior of QCD, which is Coulomb-like (throughout this paper we refer to a $\frac{1}{\ell}$ behavior as Coulombic).

The physical picture arising from this construction is as follows. The confining limit is approached as $u_{0} \rightarrow u_{\Lambda}$, then most of the string lies on the horizon ${ }^{3}$ and implies a string-like interaction between the "quarks" (the endpoints of the string) with a string tension

$$
\sigma=f\left(u_{\Lambda}\right)
$$

and a linear potential

$$
E=\sigma \cdot \ell-2 \kappa
$$

where $\kappa=\mathcal{K}\left(u_{\Lambda}\right)$ is a finite constant. On the other hand, the conformal limit is approached as $u_{0} / u_{\Lambda} \rightarrow \infty$, then the whole string is far away from the horizon and it is ruled by the geometry near the boundary. In other words, this limit correspondes in some sense to taking $u_{\Lambda}$ to zero, such that the scale parameter disappears and the conformal theory is reconstructed.

\footnotetext{
${ }^{3}$ For simplicity we depict the picture only for the case where horizon is present, but a similar picture arise also in cases with no horizon. For more extensive discussion see [16].
} 


\section{The conditions on the background to satisfy a coulombic potential at small distances}

In order to generate the QCD heavy quarks potential in an holographic model it should first reconstruct the potential at asymptotics. The conditions on the holographic background to reproduce the confining asymptotic at large distances were derived in [5]. As was discussed in the introduction, the holographic dual of the perturbative region is necessarily highly curved and hence unreliable. Nevertheless we would like to derive the conditions on the background to reproduce at small distances a potential of the form

$$
E \sim \frac{1}{\ell}
$$

The full derivation is detailed in appendix $(\mathrm{A})$.

The first step would be to expand the $f$ and $g$ functions around the boundary, which we will take to be at infinity $u_{b}=\infty$,

$$
\begin{aligned}
f(u) & =a_{k} u^{k}+\mathcal{O}\left(u^{k-1}\right) \\
g(u) & =g(\infty)+b_{j} u^{-j}+\mathcal{O}\left(u^{-j+1}\right) \\
k, j & >0
\end{aligned}
$$

We assume here that $f(u)$ diverges on the boundary as a power law and that $g(u)$ tends to a finite value on the boundary. Then we can calculate the length of the string as a function of its minimal radial position, $u_{0}$, using (2.9). Since we are interested in the small distance behavior, i.e. $u_{0}$ which is close to the boundary, the calculation will be done in the region $u_{0} \gg u_{\Lambda}$, there we find

$$
\ell\left(u_{0}\right)=\left(\frac{2 g(\infty)}{a_{k}} C_{2 k, 2 k}(\infty)\right) \frac{1}{\left(u_{0}\right)^{k-1}}+\mathcal{O}\left(\frac{1}{\left(u_{0}\right)^{k+j-1}}\right)
$$

where

$$
C_{2 k, 2 k}(\infty)=\int_{1}^{\infty} d y \frac{1}{(y)^{2 k}} \frac{1}{\sqrt{1-y^{-2 k}}}
$$

is a convergent integral. Next we calculate $\mathcal{K}\left(u_{0}\right)$ in the same manner

$$
\mathcal{K}\left(u_{0}\right)=u_{0} g(\infty)\left[1+\frac{1}{2} C_{2 k, 2 k}^{\prime}(\infty)\right]+\mathcal{O}\left(u_{0}^{1-j}\right)
$$

(where $C_{2 k, 2 k}^{\prime}(\infty)$ is another convergent integral defined in appendix $(\mathrm{A})$ ) and use (3.3) to find the inverse relation of $u_{0}$ as a function of $\ell$

$$
u_{0}=\left(\frac{2 g(\infty)}{a_{k}} C_{2 k, 2 k}(\infty) \frac{1}{\ell}\right)^{\frac{1}{k-1}}
$$


in order to express $\mathcal{K}$ as a function of the length

$$
\mathcal{K}=\left[1+\frac{1}{2} C_{2 k, 2 k}^{\prime}(\infty)\right]\left[2 C_{2 k, 2 k}(\infty)\right]^{\frac{1}{k-1}}[g(\infty)]^{\frac{k}{k-1}}\left(\frac{1}{a_{k} \ell}\right)^{\frac{1}{k-1}}
$$

Then we are ready to substitute these results into the expression for the energy of the string (2.12) as a function of its length to get

$$
\begin{aligned}
E(\ell) & =f\left(u_{0}(\ell)\right) \cdot \ell-2 \mathcal{K}(\ell) \\
& =N_{k}[g(\infty)]^{\frac{k}{k-1}}\left(\frac{1}{a_{k} \ell}\right)^{\frac{1}{k-1}}
\end{aligned}
$$

where $N_{k}$ is a $k$-dependent dimensionless number

$$
N_{k}=\left[1+2 C_{2 k, 2 k}(\infty)+\frac{1}{2} C_{2 k, 2 k}^{\prime}(\infty)\right]\left[2 C_{2 k, 2 k}(\infty)\right]^{\frac{1}{k-1}}
$$

The bottom line is that the dependence of the energy on the length in the region close to the boundary, i.e. small distances, is

$$
E \sim\left(\frac{1}{\ell}\right)^{\frac{1}{k-1}}
$$

We conclude that in order for the potential to satisfy a coulombic form near the boundary, one has to demand $k=2$. In other words, the condition on the backround is

$$
f\left(u \gg u_{\Lambda}\right) \sim u^{2}
$$

We emphasize that this analysis is valid as long as $f$ diverges on the boundary as a power law and $g$ is finite there. An example for a case that fulfills this ansatz is the famous $A d S_{5} \times S_{5}$ solution in which

$$
\begin{aligned}
& f(u)=\frac{1}{2 \pi \alpha^{\prime}}\left(\frac{u}{R_{A d S}}\right)^{2} \\
& g(u)=\frac{1}{2 \pi \alpha^{\prime}}
\end{aligned}
$$

\section{Renormalization}

Generically, the bare energy of the string in the holographic construction (2.10) is divergent. From the bulk perspective it is a result of the infiniteness of the boundary. The string's endpoints are tied to the boundary and the energetically preferred state is when the string extends to the bulk ${ }^{4}$. Since the boundary is radialy located at

\footnotetext{
${ }^{4}$ There may be gravitational solutions in which this is not the case and the energetically preferred state is when the whole string extends to the boundary only, but then the Wilson loop is just the flat space Wilson loop. We do not consider such a case as we are interested in holographic Wilson loops.
} 
infinity, this would mean that the string's length is infinite, and this is the origin of the divergence. From the field theory living on the boundary perspective the infinite bare mass of the quarks is the source for the divergence. The most simple and naive approach would be to subtract this bare mass [6], as one can think of the quark as a straight string stretched from $u=0$ (or $u=u_{\Lambda}$ if there exist an horizon at $u_{\Lambda}$ ) to $u=u_{b}^{5}$ such that its mass is given by

$$
m_{q}=\int_{u_{\Lambda}}^{u_{b}} d u g(u)
$$

That approach was taken in (2) following [5]. However, there may be other regularization schemes as well, and we want to discuss and compare them. In this section we examine two other schemes: the holographic scheme [7] and the Legendre transform scheme [8]. We calculate analytically the subtracted part of the energy in each of the schemes, and then discuss the results.

The calculation is done using the expression for the bare energy (2.10) and assuming the following assumptions on the $f$ and $g$ functions:

1. $f$ is a monotonic increasing function

2. $g$ is a constant function or a monotonic decreasing function which does not diverge on the boundary

The first one is the same as the ansatz for the $f$ function in [5], while the second ansatz is more restrictive, but in fact is not needed in our analysis of the Legendre transform scheme.

\subsection{Holographic renormaliztion}

The idea behind this scheme [7] is to expand the bare energy around the boundary, identify the infinite terms and subtract them from the original expression of the bare energy. Essentialy we remove infinities resulting from the infiniteness of the boundary. Therefore we will not lose any information about the subtracted part if we will consider the bare energy with $u_{0}$ which is far from the horizon. Then $g(u)$ is approximately constant in all the range of integration

$$
E_{\text {bare }}\left(u_{0}\right) \cong 2 g\left(u_{b}\right) \int_{u_{0}}^{u_{b}} d u \frac{1}{\sqrt{1-\left(\frac{f\left(u_{0}\right)}{f(u)}\right)^{2}}}
$$

Now let us divide the integration into two parts

$$
E_{\text {bare }}\left(u_{0}\right)=2 g\left(u_{b}\right)\left[\int_{u_{m}}^{u_{b}} d u \frac{1}{\sqrt{1-\left(\frac{f\left(u_{0}\right)}{f(u)}\right)^{2}}}+\int_{u_{0}}^{u_{m}} d u \frac{1}{\sqrt{1-\left(\frac{f\left(u_{0}\right)}{f(u)}\right)^{2}}}\right]
$$

\footnotetext{
${ }^{5}$ In this section we will keep the notation in which the boundary is located at $u=u_{b}$. Later on we will set $u_{b}=\infty$.
} 
such that $f(u) \gg f\left(u_{0}\right)$ above $u_{m}$ so we can simplify this expression

$$
\begin{aligned}
E_{\text {bare }}\left(u_{0}\right) \cong 2 g\left(u_{b}\right)\left[\int_{u_{m}}^{u_{b}} d u+\int_{u_{0}}^{u_{m}} d u \frac{1}{\sqrt{1-\left(\frac{f\left(u_{0}\right)}{f(u)}\right)^{2}}}\right] \\
=2 u_{b} g\left(u_{b}\right)-2 g\left(u_{b}\right) u_{0}\left[\frac{u_{m}}{u_{0}}-\int_{1}^{\frac{u_{m}}{u_{0}}} d y \frac{1}{\sqrt{1-\left(\frac{f\left(u_{0}\right)}{f(u)}\right)^{2}}}\right]
\end{aligned}
$$

where in the last transition we have changed the integration variable to $y=\frac{u}{u_{0}}$. The second term does not contains boundary infinities, because its only dependence on the boundary is via $g\left(u_{b}\right)$ which is finite in our ansatz. So even if the second term includes divergent parts, this scheme will not take care of it. Writing the bare energy in that form we isolate the infinity resulting from the boundary such that according to the prescription of this scheme, the subtracted part of the energy would be

$$
E_{\text {sub }}=2 u_{b} g\left(u_{b}\right)
$$

\subsection{The Legendre transform scheme}

The idea behind this scheme [8] is to consider the legendre transform of the energy as the renormalized energy

$$
E_{\text {renormalized }}=\int d x\left(\mathcal{L}-p \cdot u^{\prime}\right)
$$

such that the subtracted part of the energy is

$$
E_{\text {sub }}=\int d x\left(p \cdot u^{\prime}\right)
$$

Now, since $p$ does not depend explicitly on $x$ (see (2.4)) we can write it as

$$
E_{\text {sub }}=\int d x \frac{\partial}{\partial x}(p \cdot u)
$$

which means that the subtracted part of the energy is a boundary term, and therefore we can expect to have a similar result as in the holographic scheme. Changing variables from $x$ to $u\left(d x \frac{\partial}{\partial x}=d u \frac{\partial}{\partial u}\right)$ and substitute the momentum (2.4) on the geodesic line (2.7), we have

$$
\begin{aligned}
E_{s u b} & =\int_{u_{0}}^{u_{b}} d u \frac{\partial}{\partial u}\left[2 u g(u) \sqrt{1-\left(\frac{f\left(u_{0}\right)}{f(u)}\right)^{2}}\right] \\
& =2 u_{b} g\left(u_{b}\right) \sqrt{1-\left(\frac{f\left(u_{0}\right)}{f\left(u_{b}\right)}\right)^{2}} \\
& =2 u_{b} g\left(u_{b}\right)
\end{aligned}
$$


which indeed result as the holographic scheme. Pay special attention to the fact that we have not used here the ansatz for the $g$ function stated in the beginning of this section and still got the same result as for the holographic scheme.

\subsection{The mass subtraction scheme}

This scheme suggests to simply subtract the bare mass of two free quarks, each of them is represented by a straight string stretched from the boundary to the horizon. Therefore the subtracted energy is given by [5]

$$
E_{s u b}=2 \int_{u_{\Lambda}}^{u_{b}} d u g(u)
$$

Let us seperate the integration into two regions

$$
E_{\text {sub }}=2 \int_{u_{m}}^{u_{b}} d u g(u)+2 \int_{u_{\Lambda}}^{u_{m}} d u g(u)
$$

where above $u_{m}, g$ is approximately stabilized on its boundary value

$$
\begin{aligned}
E_{\text {sub }} & \cong 2 g\left(u_{b}\right) \int_{u_{m}}^{u_{b}} d u+2 \int_{u_{\Lambda}}^{u_{m}} d u g(u) \\
& =2 u_{b} g\left(u_{b}\right)-u_{\Lambda}\left[2 \frac{u_{m}}{u_{\Lambda}} g\left(u_{b}\right)-2 \int_{1}^{\frac{u_{m}}{u_{\Lambda}}} d y g(y)\right]
\end{aligned}
$$

where in the last transition we have changed the integration variable to $y=\frac{u}{u_{\Lambda}}$. The first term is essentialy the same one as of the two other schemes, and is a result of the infiniteness of the boundary. The second term is a feature of the horizon and it is finite since it does not contain boundary infinities (because we isolated them) and its only dependence is on the horizon characteristics, which cannot cause a divergence of the energy (because the curvature is not singular). Its exact value can be calculated for a given model. We conclude that the subtracted energy in this scheme takes the form

$$
E_{\text {sub }}=2 u_{b} g\left(u_{b}\right)-A \cdot u_{\Lambda}
$$

where $A$ is a constant finite number that depends on the exact form of the horizon

$$
A=\lim _{\frac{u_{m}}{u_{\Lambda}} \rightarrow \infty}\left[2 \frac{u_{m}}{u_{\Lambda}} g\left(u_{b}\right)-2 \int_{1}^{\frac{u_{m}}{u_{\Lambda}}} d y g(y)\right]
$$

In the non critical $A d S_{n+1}$ solutions that will be discussed later, for example, one finds

$$
A(n)=\frac{\Gamma\left[\frac{n-1}{n}\right]}{\sqrt{\pi} \Gamma\left[\frac{n-2}{2 n}\right]}
$$



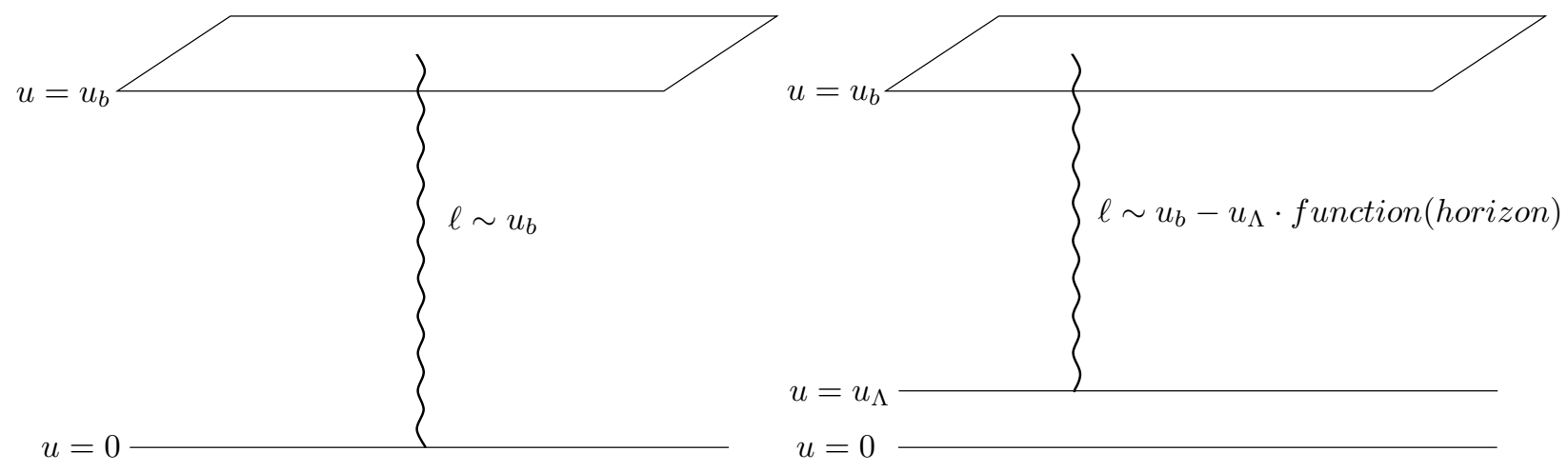

Figure 2. Left: In the case in which the renormalization scheme ignores the horizon, one can think of this scheme as of subtracting the length of a string streched from the boundary to the end of space at $u=0$, which is proportional to the location of the horizon, $u_{b}$. Right: In the mass subtraction scheme one subtracts the length of a string streched from the boundary to the horizon, which is shorter by a finite amount from the string of the first case, as the effect of the horizon must be finite. On dimensional grounds, this finite difference must be proportional to $u_{\Lambda}$ and it depends only on the characteristics of the horizon (function (horizon) is a finite dimensionless number).

\subsection{Conclusions}

We see that the mass subtraction scheme differs from the two other schemes by a constant term in the expression for the subtracted energy. The fact that different schemes differ only by a finite term is true in field theory and here we have a geometrical realization for that feature, as explained below.

The holographic and Legendre schemes are taking care only of the infintiness of the boundary, and do not take into account the existence of the horizon, while the mass subtraction scheme does take it into account. Indeed, in cases with no horizon we set $u_{\Lambda}$ to zero (according to the prescription in section (2)) and the difference between the schemes disappears. As was mentioned in the beginning of this section, the divergence is a result of the infiniteness of the boundary. The difference between the schemes is only the horizon, which cannot cause any divergences of the energy since the curvature is not singular. Therefore we conclude that the difference between the schemes must be realized as a finite affect.

An important implication of this conclusion is that the analysis of the behavior of the Wilson loop at asymptotic regions (in [5] for the confining region and in section (3) for the coulombic region) is independent of the regularization scheme (as far as those three schemes are considered).

Furthermore, out of the fact that the $U V$ region is eventually decoupled from the $I R$ region as we take the limit $u_{b} \rightarrow \infty$, one can restrict the form of the subtracted parts. Each of those regions has its own scale parameter ( $u_{b}$ and $u_{\Lambda}$, respectively) and therefore properties of each of them would depend only on the relevant scale. 
The divergent part of the energy is a feature of the $U V$ region (since the divergency is a result only of the infiniteness of the boundary) such that on dimensional grounds it would be proportional to the location of the boundary, $u_{b}$. On the other hand, the finite difference between the schemes would be proportional to $u_{\Lambda}$ since it is a result of the $I R$ properties of the background. We describe this picture in figure (2).

\section{The Wilson line of the holographic models}

So far we have made a general discussion about the Wilson line in holographic backgrounds. As we aim to compare the QCD Wilson line to the one of holographic theories, we have to consider specific models. In this section we briefly review few representative holographic models, which we latter compare to lattice simulations, and discuss their Wilson line. Among the large class of holographic models we have choose to consider the near extremal $D_{3}$ and $D_{4}$ branes, non critical $A d S_{6}$ and the Klebanov Strassler construction. The reason for this choice is that these backgrounds admit a Wilson line which is in principle close to the QCD Wilson line (at least on asymptotics), as we will discuss below. In addition, they are all solutions of string theory or of supergravity. Other examples have been analyzed in the literature, among them also phenomenological models and backgrounds which are not solutions of a particular action. In this paper we choose not to deal with such cases since one can always engineer a background which will admit some required features without being a solution of an action. Instead, we want to ask what is the solution that admits a Wilson line which is closest to the QCD Wilson line. Nevertheless, for a matter of completeness, we shortly review several other results; The Wilson line of the phenomenological Holographic QCD [17] has been analyzed in [18] where a good agreement to the lattice results was found. In [19], the Wilson line of some hard-wall scenario was calculated and it was found to admit a Cornell potential form ${ }^{6}$.

Near extremal $D_{p}$ brane

In the supergravity background associated to near extremal $D_{p}$ brane [20] one finds

$$
\begin{aligned}
f^{2}(u) & =\left(\frac{1}{2 \pi \alpha^{\prime}}\right)^{2}\left(\frac{u}{R_{A d S}}\right)^{7-p} \\
g^{2}(u) & =\left(\frac{1}{2 \pi \alpha^{\prime}}\right)^{2} \frac{1}{1-\left(\frac{u_{\Lambda}}{u}\right)^{7-p}}
\end{aligned}
$$

These backgrounds satisfy the condition for confinement for all values of $p$. On the other hand, using the analysis of (3), we see that the potential in the small distances region takes the form

$$
E \sim\left(\frac{1}{\ell}\right)^{\frac{2}{5-p}}
$$

\footnotetext{
${ }^{6}$ We thank Andrew Zayakin for referring us to this paper.
} 
Therefore, only for $p=3$ the potential behaves as $\frac{1}{\ell}$ in the small distances region. In that case there are four coordinates on the boundary, where one of them is compactified. For that reason, on energy scales smaller than one over the compactification radius

$$
E \lesssim \frac{1}{R_{\text {compactification }}}
$$

the theory on the boundary is effectively three dimensional. The conclusion is that the field theory on the boundary of the near extremal $D_{3}$ brane is four dimensional at small lengths scale (or equivalently high energies scale), where the potential is Coulombic, but it is effectively three dimensional on large lengths scale (low energies), where the potential is confining. In order to finish with an effectively four dimensional boundary at large distances, one needs to consider the near extremal $D_{4}$ brane, following the same argument. In that case the boundary theory on small length scales would be five dimensional and the potential there will take the form $\sim \frac{1}{\ell^{2}}$.

\section{Non critical $A d S$ solutions}

The metric of a non-critical $A d S_{n+1}$ solution of type II string theory $[11,12]$ with one of the spatial coordinates compactified on a circle is given by

$$
\begin{aligned}
d s^{2} & =\frac{1}{2 \pi \alpha^{\prime}}\left(\frac{u}{R_{A d S}}\right)^{2}\left[-d t^{2}+d x_{i}^{2}+\left(1-\left(\frac{u_{\Lambda}}{u}\right)^{n}\right) d x_{\text {comp }}^{2}\right]+\frac{1}{2 \pi \alpha^{\prime}}\left(\frac{R_{A d S}}{u}\right)^{2} \frac{d u^{2}}{\left(1-\left(\frac{u_{\Lambda}}{u}\right)^{n}\right)} \\
i & =1, \ldots,(n-2)
\end{aligned}
$$

where $u_{\Lambda}^{n}$ is the energy density on the brane. Therefore the $f$ and $g$ functions take the following forms

$$
\begin{aligned}
& f^{2}(u)=\frac{1}{\left(2 \pi \alpha^{\prime}\right)^{2}}\left(\frac{u}{R_{A d S}}\right)^{4} \\
& g^{2}(u)=\frac{1}{\left(2 \pi \alpha^{\prime}\right)^{2}} \frac{1}{1-\left(\frac{u_{\Lambda}}{u}\right)^{n}}
\end{aligned}
$$

This background satisfies both conditions for confinement at long distances and $\frac{1}{\ell}$ potential at short distances, for any value of $n$ (larger than 2). For these conditions we have to add the demand that the effective boundary theory at long distances would be four dimensional. Therefore, a good candidate would be the non-critical $A d S_{6}$ which has a five dimensional boundary with one compactified coordinate (and hence a four dimensional effective field theory on the boundary, following a similar argument as in the D branes discussion).

\section{Klebanov Strassler}

The supergravity solution of the deformed conifold is of the following form $[13,21]$

$$
d s^{2}=h^{-\frac{1}{2}}(\tau) d x_{0123}^{2}+h^{\frac{1}{2}}(\tau) d s_{6}^{2}
$$


where $d s_{6}^{2}$ is the metric of the deformed conifold

$d s_{6}^{2}=\frac{\epsilon^{4 / 3}}{2} K(\tau)\left[\frac{1}{3 K^{3}(\tau)}\left(d \tau^{2}+\left(g^{5}\right)^{2}\right)+\cosh ^{2}\left(\frac{\tau}{2}\right)\left[\left(g^{3}\right)^{2}+\left(g^{4}\right)^{2}\right]+\sinh ^{2}\left(\frac{\tau}{2}\right)\left[\left(g^{1}\right)^{2}+\left(g^{2}\right)^{2}\right]\right]$

$\epsilon$ is the energy scale and the functions $h(\tau)$ and $K(\tau)$ are given by

$$
\begin{gathered}
h(\tau)=\left(g_{s} M \alpha^{\prime}\right)^{2} 2^{2 / 3} \epsilon^{-8 / 3} I(\tau) \\
I(\tau) \equiv \int_{\tau}^{\infty} d x \frac{x \operatorname{coth} x-1}{\sinh ^{2} x}(\sinh 2 x-2 x)^{\frac{1}{3}} \\
K(\tau)=\frac{(\sinh (2 \tau)-2 \tau)^{\frac{1}{3}}}{2^{\frac{1}{3}} \sinh \tau}
\end{gathered}
$$

$\tau$ is the radial coordinate, it is a pure numer (i.e. unitless) running from zero to infinity on the boundary. Near the boundary, i.e. at large $\tau$, the $h(\tau)$ and $K(\tau)$ function takes the form

$$
\begin{gathered}
h(\tau \gg 1)=\left(g_{s} M \alpha^{\prime}\right)^{2} 3 \cdot 2^{2 / 3} \epsilon^{-8 / 3} \tau e^{-\frac{4}{3} \tau} \\
K(\tau \gg 1)=2^{\frac{1}{3}} e^{-\frac{1}{3} \tau}
\end{gathered}
$$

From the form of the metric we conclude that the $f$ and $g$ functions are given by

$$
\begin{aligned}
& f^{2}(\tau)=h^{-1}(\tau) \\
& g^{2}(\tau)=\frac{\epsilon^{4 / 3}}{6} \frac{1}{K^{2}(\tau)}
\end{aligned}
$$

Following [21] one can check that the KS background is confining since $f(\tau)$ has a minimum at $\tau=0$ and it is finite there $f(0) \neq 0$. On the other hand, near the boundary $(\tau \gg 0)$ this backgroud do not follows the form (3.2) required for the analysis in section (3). However, it is approximately $A d S$ in this region, therefore we expect it to generate a potential which is approximately $\frac{1}{\ell}$ at short distances. Near the boundary the KS metric takes the form

$$
d s^{2} \sim \epsilon^{4 / 3} \tau^{-\frac{1}{2}} e^{\frac{2}{3} \tau} d x_{0123}^{2}+\tau^{\frac{1}{2}} d \tau^{2}+\ldots
$$

The behavior of the wrap factors is dominated by the exponential term. Therefore, by neglecting the power law terms of the wrap factors we change the geometry just by little. The resultant approximate geometry is then

$$
d s^{2} \sim \epsilon^{4 / 3} e^{\frac{2}{3} \tau} d x_{0123}^{2}+d \tau^{2}+\ldots
$$

which can be brought, by an appropriate coordinate redefinition, to the form of an AdS geometry

$$
d s^{2} \sim\left(\frac{u}{R}\right)^{2} d x_{0123}^{2}+\left(\frac{R}{u}\right)^{2} d u^{2}+\ldots
$$


Hence, since the $A d S$ geometry generates a $\frac{1}{\ell}$ potential, we expect the KS background to generate a potential which is approximately $\frac{1}{\ell}$ near the boundary. Then the arising question is how do the corrections to the approximate $\frac{1}{\ell}$ potential look like? To answer this question we use the theorem reviewed in (2) (the exact analysis is detailed in appendix $(\mathrm{G})$ ). Plugging the discussed geometry (5.13) into the expression for the length of the string (2.9) one finds

$$
\ell\left(\tau_{0}\right) \sim \epsilon^{2 / 3} \frac{1}{f\left(\tau_{0}\right)} e^{+\frac{1}{3} \tau_{0}} \sim \frac{g_{s} M \alpha^{\prime}}{\epsilon^{2 / 3}} \tau_{0}^{\frac{1}{2}} e^{-\frac{1}{3} \tau_{0}}
$$

In the same way the Kappa function (2.13) takes the form

$$
\mathcal{K}\left(\tau_{0}\right) \sim \epsilon^{2 / 3} e^{\frac{1}{3} \tau_{0}}
$$

Then the energy of the string (2.12) would be

$$
E\left(\tau_{0}\right) \sim-\epsilon^{2 / 3} e^{\frac{1}{3} \tau_{0}}
$$

In order to derive the dependence of the energy on the length we need to invert the function $\ell\left(\tau_{0}\right)$ into $\tau_{0}(\ell)$ and plug it into the expression for the energy $E(\ell) \sim$ $-\epsilon^{2 / 3} e^{\frac{1}{3} \tau_{0}(\ell)}$. This is very difficult to perform. Instead we will expand $\ell\left(\tau_{0}\right)$ around large $\tau_{0}$ and then invert the relation. For that it would be usefull to take the logarithm of the length

$$
\ln \left[\left(g_{s} M \alpha^{\prime}\right)^{-1} \epsilon^{2 / 3} \ell\right] \sim-\frac{1}{3} \tau_{0}+\ln \sqrt{\tau_{0}}
$$

The leading order

$$
\ln \left[\left(g_{s} M \alpha^{\prime}\right)^{-1} \epsilon^{2 / 3} \ell\right] \sim-\frac{1}{3} \tau_{0}
$$

would result in the leading $\frac{1}{\ell}$ behavior

$$
E \sim-\frac{g_{s} M \alpha^{\prime}}{\ell}
$$

as expected. In order to calculate the leading correction we would plug the leading order relation (5.20) into the subleading term of the exact expression (5.19)

$$
\ln \left[\left(g_{s} M \alpha^{\prime}\right)^{-1} \epsilon^{2 / 3} \ell\right] \sim-\frac{1}{3} \tau_{0}+\ln \sqrt{-\ln \left[\left(g_{s} M \alpha^{\prime}\right)^{-1} \epsilon^{2 / 3} \ell\right]}
$$

to get the dependence of $\tau_{0}$ on $\ell$

$$
\frac{1}{3} \tau_{0} \sim-\ln \left[\left(g_{s} M \alpha^{\prime}\right)^{-1} \epsilon^{2 / 3} \ell\right]+\ln \sqrt{-\ln \left[\left(g_{s} M \alpha^{\prime}\right)^{-1} \epsilon^{2 / 3} \ell\right]}
$$

Inserting this into the expression for the energy we finish with a potential of the form

$$
E(\ell) \sim-\frac{\left(g_{s} M \alpha^{\prime}\right) \sqrt{-\ln \left[\left(g_{s} M \alpha^{\prime}\right)^{-1} \epsilon^{2 / 3} \ell\right]}}{\ell}
$$

In principle, there could appear also a constant term, as will be discussed in section (8). In appendix $(G)$ we explicitly calculate it. 


\section{Units of measurement}

In order to make a quantitative comparison between different models one has to discuss the issue of units of measurement. In this section we discuss two issues concerning this subject. We start with an abstract discussion and then specialize to the models under investigation.

The first issue is the question of how to determine the system of units when infinitely distant scales are introduced. In a theory with only one scale parameter (say, the fundamental string length $l_{s}$ ) every physical quantity $Q$ will be expressed as some power of the only scale

$$
Q \sim\left(l_{s}\right)^{p}
$$

Now consider the case in which another scale $R$ is introduced. When the two scales are very far apart $Q$ would have to be expressed as some powers of both

$$
Q \sim\left(l_{s}\right)^{p_{1}}(R)^{p_{2}}
$$

If we further seperate the two scales by an infinite gap, there would be a unique choice of the powers $p_{1}$ and $p_{2}$ such as to get finite answers, and this is the way one has to choose his system of units. Any other system would make no sense since it would result with infinite values for physical quantities.

The second question is how to compare different models which have different scale parameters. Each model has its own scale parameters and therefore the immediate system of units is different in each of them. In order to quantitatively compare different models one has to describe the quantities under comparison in a model-independent way, i.e. they have to be expressed in terms of universal physical quantities that are present in all the models under investigation.

Specializing to the framework we explore in this paper, the physical quantities we are interested in are the energy of the string and the distance between its endpoints on the boundary, the scales are the fundamental string length $l_{s}$ and the radius of the space $R_{\text {space }}$, and the fact that the two scales are infinitely far away from each other is exactly the statement of weakly curved holography

$$
\alpha^{\prime}=l_{s}^{2} \ll R_{\text {space }}^{2}
$$

It is clear now that since $l_{s}$ and $R_{\text {space }}$ do not exist in the lexicon of the gauge theory, we need to express the energy and length in terms of universal physical quantities which are present in both frameworks, stringy holography and gauge theory. Since we are dealing with aspects of strong interaction, the natural universal physical quantities are the string tension and the glueball mass (because they must exist in any model that aims to describe strong interactions). In this language, the SUGRA limit is

$$
\sigma \gg m_{\text {glueball }}^{2}
$$


Being expressed in such a way, we would be able to compare the Wilson line of these two frameworks on equal footing.

As a realization of this general discussion, we first consider the near extremal $D_{p}$ brane. In appendix (C) we apply the theorem presented in section (2) to find the expressions for the energy and length of the string in this case. We find that the energy of a classical string is well-defined when it is measured in units of

$$
[E]=\frac{u_{\Lambda}}{\alpha^{\prime}}
$$

where $u_{\Lambda}$ is the scale parameter and $\alpha^{\prime}$ is related to the fundamental string length. The length of a classical string is well-defined when it is measured in units of

$$
[\ell]=u_{\Lambda}\left(\frac{R_{A d S}}{u_{\Lambda}}\right)^{\frac{7-p}{2}}
$$

where $R_{A d S}$ is the radius of the $A d S$ space and is of the same order as $u_{\Lambda}$. In this system of units, following equation (2.14), the string tension will take the value

$$
\sigma=\frac{1}{2 \pi}
$$

in units of (the units of the string tension are essentially the units of the energy over the units of length)

$$
[\sigma]=\frac{[E]}{[\ell]}=\frac{1}{\alpha^{\prime}}\left(\frac{u_{\Lambda}}{R_{A d S}}\right)^{\frac{7-p}{2}}
$$

Any other choice of system would result in an infinite value for the string tension in the SUGRA limit. The glueball mass scale is related to the radius of the compactified coordinate

$$
m_{g b} \sim \frac{1}{R_{x_{\text {compactified }}}}
$$

which is uniquely determined from the demand of avoiding a conical singularity. In appendix (B) we review this calculation and show that in the case of the near extremal $D_{p}$ brane background it is given by

$$
2 \pi R_{x_{\text {compactified }}}=\frac{4 \pi}{7-p} u_{\Lambda}\left(\frac{u_{\Lambda}}{R_{A d S}}\right)^{-\frac{7-p}{2}}
$$

Then the glueball mass is

$$
m_{g b} \sim \frac{1}{u_{\Lambda}}\left(\frac{u_{\Lambda}}{R_{A d S}}\right)^{\frac{7-p}{2}}
$$

The relation between the string tension $\left(\sigma=\frac{1}{2 \pi} \frac{1}{\alpha^{\prime}}\left(\frac{u_{\Lambda}}{R_{A d S}}\right)^{\frac{7-p}{2}}\right)$ and the glueball mass would take the form

$$
m_{g b} \sim \frac{\alpha^{\prime}}{u_{\Lambda}} \sigma
$$


Now we can express the energy and length in terms of $\sigma$ and $m_{g b}$

$$
\begin{gathered}
{[E]=\frac{u_{\Lambda}}{\alpha^{\prime}} \sim \frac{\sigma}{m_{g b}}} \\
{[\ell]=u_{\Lambda}\left(\frac{R_{A d S}}{u_{\Lambda}}\right)^{\frac{7-p}{2}} \sim \frac{1}{m_{g b}}}
\end{gathered}
$$

Being expressed in such a way, the energy and length are well-defined and their values on both sides (holography and gauge theory) are compareable (given that they are expressed in a similar way in the gauge theory). The exact translation is derived in the next section.

For the case of non-critical $A d S_{n+1}$ a similar analysis holds. The string tension is given by

$$
\sigma=\frac{1}{2 \pi \alpha^{\prime}}\left(\frac{u_{\Lambda}}{R_{A d S}}\right)^{2}
$$

As the periodicity of the compactified dimension is given by $\beta \equiv 2 \pi R_{A d S}=\frac{4 \pi}{n} \frac{R^{2}}{u_{\Lambda}}$ (see appendix (B)), the glueball mass would be

$$
m_{g b} \sim \frac{u_{\Lambda}}{R_{A d S}^{2}}
$$

and the same relation (6.10) between $m_{g b}$ and $\sigma$ holds. Using the appropriate units for the energy and length found in appendix (E) we reconstruct (6.11) and (6.12) for the present case

$$
\begin{gathered}
{[E]=\frac{u_{\Lambda}}{\alpha^{\prime}} \sim \frac{\sigma}{m_{g b}}} \\
{[\ell]=\frac{R_{A d S}^{2}}{u_{\Lambda}} \sim \frac{1}{m_{g b}}}
\end{gathered}
$$

Next we consider the Klebanov Strassler construction. The string tension is given by $(2.14)$

$$
\sigma=f(0)=h^{-\frac{1}{2}}(0)=2^{-1 / 3} I(0) \frac{\epsilon^{4 / 3}}{g_{s} M \alpha^{\prime}} \cong 0.57 \frac{\epsilon^{4 / 3}}{g_{s} M \alpha^{\prime}}
$$

and the glueball mass is [13]

$$
m_{g b} \sim \frac{\epsilon^{2 / 3}}{g_{s} M \alpha^{\prime}}
$$

where $\epsilon$ is the scale parameter and $g_{s}$ is the fundamental string coupling and $M$ is the rank of the symmetry group. Using the form of the metric (5.5) one can find the appropriate units for the energy and length of the string ${ }^{7}$ and express them in terms of the string tension and the glueball mass

\footnotetext{
${ }^{7}$ Since the radial coordinate is dimensionless, the units of the energy are essentially the units of the $g$ function whereas the units of the length are the units of the $g$ function over the units of the $f$ function.
} 


\begin{tabular}{|c|c|c|}
\hline & $\sigma$ & $m_{g b}=m_{0^{++}}$ \\
\hline \hline$D_{3}$ brane & $\frac{1}{2 \pi \alpha^{\prime}}\left(\frac{u_{\Lambda}}{R_{A d S}}\right)^{2}$ & $\sqrt{11.59} \frac{u_{\Lambda}}{R_{A d S}^{2}} \cong 3.4 \frac{u_{\Lambda}}{R_{A d S}^{2}}$ \\
\hline$D_{4}$ brane & $\frac{1}{2 \pi \alpha^{\prime}}\left(\frac{u_{\Lambda}}{R_{A d S}}\right)^{\frac{3}{2}}$ & $\left(9.39 \cdot \frac{3}{4 \pi}\right) \frac{U_{\Lambda}^{1 / 2}}{R_{A d S}^{3 / 2}} \cong 2.24 \frac{U_{\Lambda}^{1 / 2}}{R_{A d S}^{3 / 2}}$ \\
\hline$A d S_{6}$ & $\frac{1}{2 \pi \alpha^{\prime}}\left(\frac{u_{\Lambda}}{R_{A d S}}\right)^{2}$ & $\left(6.34 \cdot \frac{5}{4 \pi}\right) \frac{u_{\Lambda}}{R_{A d S}^{2}} \cong 2.52 \frac{u_{\Lambda}}{R_{A d S}^{2}}$ \\
\hline$K S$ & $0.57 \frac{\epsilon^{4 / 3}}{g_{s} M \alpha^{\prime}}$ & $0.86 \frac{\epsilon^{2 / 3}}{g_{s} M \alpha^{\prime}}$ \\
\hline
\end{tabular}

Table 1. String tension and lowest $0^{++}$glueball mass of the various holographic models.

$$
\begin{gathered}
{[E]=\epsilon^{2 / 3} \sim \frac{\sigma}{m_{g b}}} \\
{[\ell]=\frac{g_{s} M \alpha^{\prime}}{\epsilon^{2 / 3}} \sim \frac{1}{m_{g b}}}
\end{gathered}
$$

The exact translations between the natural system of units of each holographic model to the universal one is derived in the next section.

\section{Comparison with lattice gauge theories}

In this section we will compare the potential of the holographic models to lattice results of $S U(2)$ and $S U(3)$ gauge theories without fermions [22, 23]. In order to do so we first have to express everything in terms of universal physical quantities, as described in section (6). Our next task would be to determine the exact translation between the natural system of units of each holographic model to the universal one. In table (1) we summarize the results for the string tension and the lowest $0^{++}$glueball mass ${ }^{8}$ of the various holographic models. The lowest $0^{++}$glueball mass for the $D_{3}, D_{4}, A d S_{6}$ and $K S$ models was calculated in [24], [25], [12] and [26], respectively. The units of [26]'s results are explicitly resolved in [27]. Then it is simple to invert the relations such as to result with a dictionary of translation between the natural system of units of each holographic model to the universal one, see table (2).

The next step would be to express the system of units used by the lattice conventions in terms of the universal physical quantities discussed above. In the lattice

\footnotetext{
${ }^{8}$ The reason for choosing the $0^{++}$state for the universal system of units is that in $S U(N)$ gauge theory it has the lowest mass.
} 


\begin{tabular}{|c|c|c|}
\hline & {$[E]$} & {$[\ell]$} \\
\hline \hline$D_{3}$ brane & $\frac{u_{\Lambda}}{\alpha^{\prime}}=(3.4 \cdot 2 \pi) \frac{\sigma}{m_{g b}}$ & $\frac{R_{A d S}^{2}}{u_{\Lambda}}=3.4 \frac{1}{m_{g b}}$ \\
\hline$D_{4}$ brane & $\frac{u_{\Lambda}}{\alpha^{\prime}}=(2.24 \cdot 2 \pi) \frac{\sigma}{m_{g b}}$ & $\frac{R_{A d S}^{3 / 2}}{u_{\Lambda}^{1 / 2}}=2.24 \frac{1}{m_{g b}}$ \\
\hline$A d S_{6}$ & $\frac{u_{\Lambda}}{\alpha^{\prime}}=(2.52 \cdot 2 \pi) \frac{\sigma}{m_{g b}}$ & $\frac{R_{A d S}^{2}}{u_{\Lambda}}=2.52 \frac{1}{m_{g b}}$ \\
\hline$K S$ & $\epsilon^{2 / 3}=1.51 \frac{\sigma}{m_{g b}}$ & $\frac{g_{s} M \alpha^{\prime}}{\epsilon^{2 / 3}}=0.86 \frac{1}{m_{g b}}$ \\
\hline
\end{tabular}

Table 2. Dictionary of translation between the natural system of units of each holographic model to the universal one.

conventions, length is measured in units $r_{0}$ related to the string tension via the next expression [23]

$$
\sigma r_{0}^{2}=1.65-\frac{\pi}{12}
$$

The value of the lowest $0^{++}$glueball mass in $D=3+1$ dimensions is given by [28]

$$
\begin{gathered}
m_{\text {glueball }} \equiv m_{0^{++}}=d_{N} \cdot \sqrt{\sigma} \\
d_{N}= \begin{cases}3.87 & N=2 \\
3.65 & N=3\end{cases}
\end{gathered}
$$

Hence we can deduce the relation between the original lattice length's unit to the desired ones

$$
r_{0}=d_{N} \sqrt{1.65-\frac{\pi}{12}} \frac{1}{m_{g b}}
$$

In the same manner the translation of the energy units, which are given in the lattice convention by $\frac{1}{r_{0}}$, is done using the next expression

$$
\frac{1}{r_{0}}=\frac{d_{N}}{\sqrt{1.65-\frac{\pi}{12}}} \frac{\sigma}{m_{g b}}
$$

The exact expressions for the potentials in $D_{p}$ brane, non-critical $A d S_{n+1}$ and Klebanov-Strassler backgrounds are derived explicilty in appendices (C), (E) and $(\mathrm{G})$, respectively. They are presented together with the lattice data in terms of the universal system of units and using two different conventions

1. Fixing the zero energy point (as in the conventions of [23]).

2. Comparing the confining asymptotic behavior of all the graphs.

in figures (3) and (4), respectively. 


\section{Heavy Quarks Potential - Fixed Zero Energy Point}

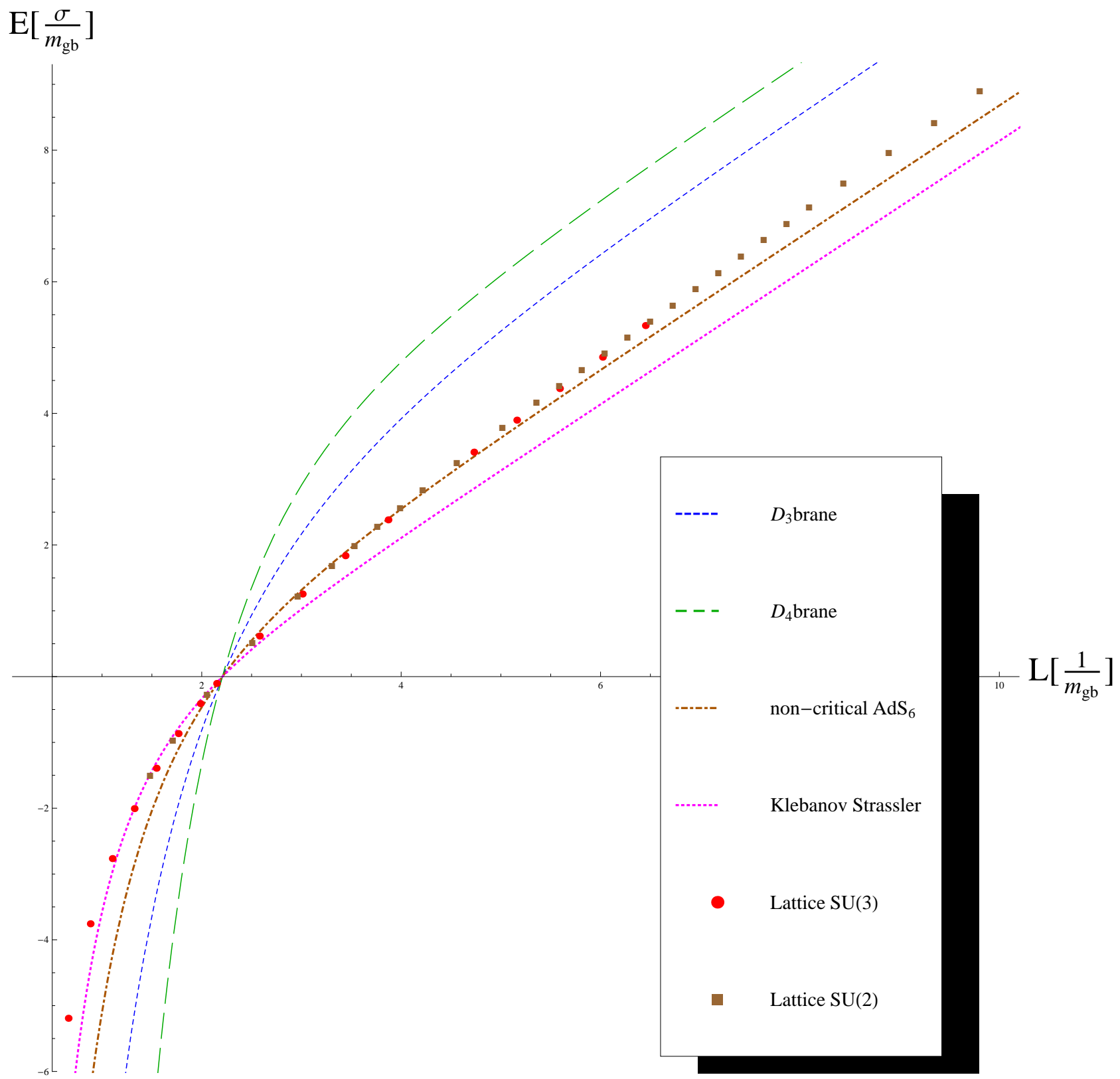

Figure 3. The heavy quarks potential of the holographic models and the lattice data expressed in the appropriate physical units and presented using the fixed zero energy point convention. 


\section{Heavy Quarks Potential - Unified Confining Asymptotic Behavior}

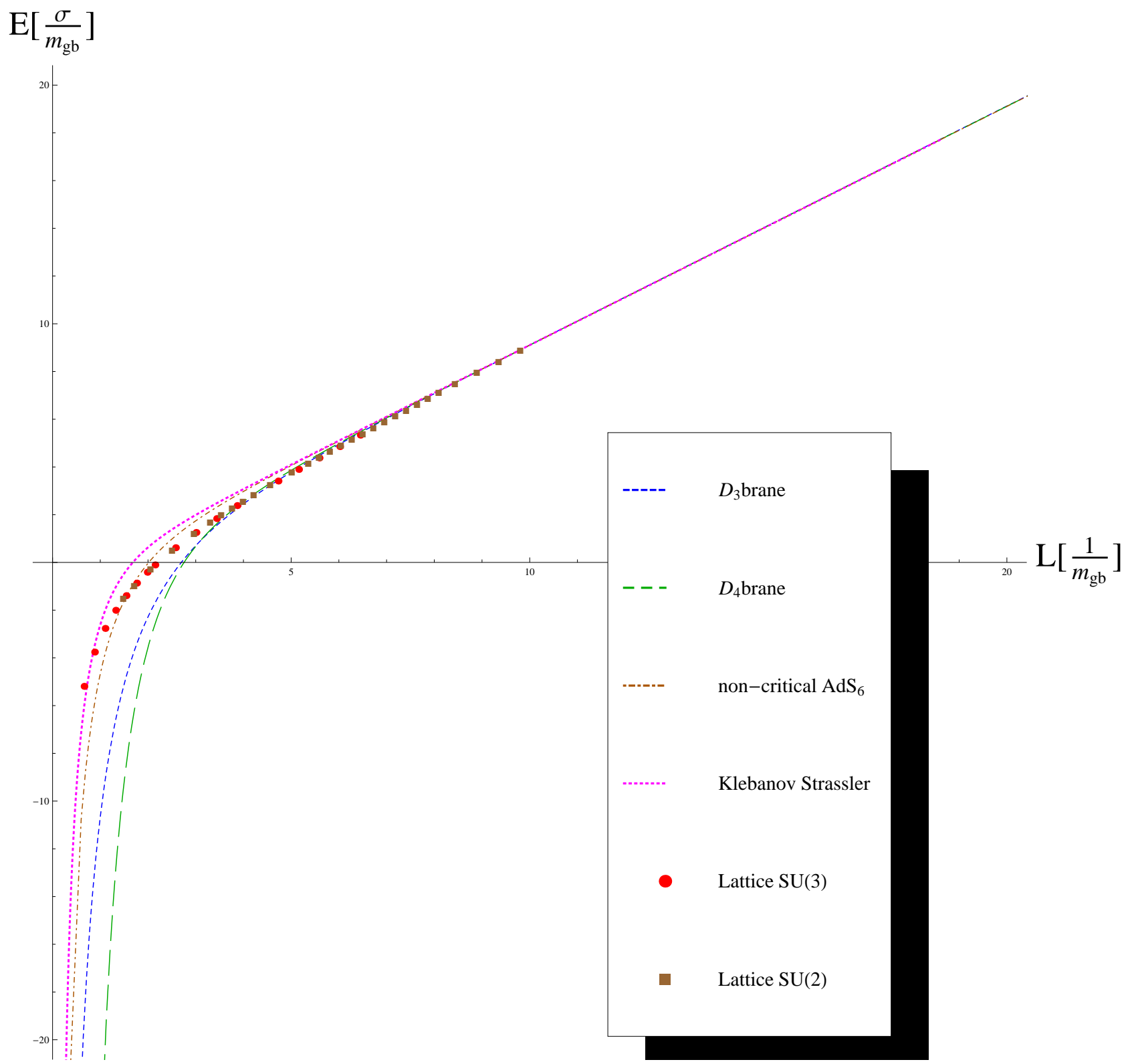

Figure 4. The heavy quarks potential of the holographic models and the lattice data expressed in the appropriate physical units and presented using the convention in which we compare the confining asymptotic behavior of all the graphs. 


\begin{tabular}{|c|c|c|c|}
\hline Case $\downarrow$ & $\begin{array}{c}\text { Fixed zero } \\
\text { energy point }\end{array}$ & Best fit & $\begin{array}{c}\text { Unified confining } \\
\text { asymptotic behavior }\end{array}$ \\
\hline \hline near extremal $D_{3}$ brane & 24.6 & 24.4 & 34.8 \\
\hline near extremal $D_{4}$ brane & 108 & 108 & 120 \\
\hline non critical $A d S_{6}$ & 4.14 & 3.98 & 5.89 \\
\hline Klebanov Strassler & 1.4 & 1.33 & 14.5 \\
\hline
\end{tabular}

Table 3. $\chi^{2}$ values for the measure of correspondence between the holographic models' potential and the lattice measurements for $\mathrm{SU}(3)$. The calculation was done using three different conventions, as detailed in the text.

In order to quantify the measure of correspondence of the holographic models' potential to the lattice data we will use Pearson's $\chi^{2}$ test

$$
\chi^{2}=\sum_{i=1}^{n} \frac{\left(O_{i}-E_{i}\right)^{2}}{\left|E_{i}\right|}
$$

where $n$ is the number of the lattice data points. The computation is done using the two conventions described above and in addition we present another convention in which the height of the theoretical potential curve is adjusted to result with a minimal $\chi^{2}$, i.e. the best fit possible. We calculate $\chi^{2}$ only for the correspondence with the SU(3) simulation ${ }^{9}$. The results are presented in table (3).

From the results we can determine that two of the models we have examined correspond significantly better to the lattice measurement of the Wilson line than the two other ones. The good models are the Klebanov Strassler and the noncritical $A d S_{6}$ backgrounds which have significantly much better $\chi^{2}$ values than the near extremal $\mathrm{D}$ branes. On the other hand, the $D_{4}$ brane background generates a potential which do not fit at all to the lattice potential, with $\chi^{2}$ 's greater than 100 for all the conventions of comparison we have used.

Out of this summary we can already conclude that the requirement for a Coulombic behavior at short distances proves itself in the sense that the models which admit at short distances a potential which is either exactly or approximately of the form $\sim \frac{1}{\ell}$ are also in a good agreement with the lattice measurements, compared to the $D_{4}$ brane which admits a $\sim \frac{1}{\ell^{2}}$ potential in this regime and has a $\chi^{2}$ value which is far above all the other. Hence it makes sense to use this requirement when searching for an holographic model which mimics QCD's Wilson line.

The best fit convention of comparison gives almost identical results to the fixed zero energy point convention. In both, the model which has the best correspondence

\footnotetext{
${ }^{9}$ We read [22]'s results for the $\mathrm{SU}(2)$ simulation out of the graph since they are not given explicitly, therefore they will be used only for a qualitative picture and not for quantitive measures
} 
with the lattice results is the $K S$ with a remarkable $\chi$ square value, but nevertheless the $A d S_{6}$ also fits quite well. In the third convention (unified confining asymptotic behavior) they flip over as the $K S$ becomes secondary to the $A d S_{6}$ which best fits.

\section{The constant terms}

At the beginning of this paper we have made a statement that at large distances the potential is linear and at small distances it is Coulombic. However, there is still a possibility of an additional constant term for each of these asymptotic behaviors, i.e.

$$
\begin{gathered}
E_{\text {long }}=\sigma \ell+C_{L} \\
E_{\text {short }} \sim-\frac{1}{\ell}+C_{S}
\end{gathered}
$$

In a sense, these constant terms characterize the behavior of the potential at intermediate distances. Therefore, a comparison between such constants of the holographic models to the constants of the gauge theory would constitute a measure for the similarity of the potentials at intermediates.

In principle, each of the constants $\left(C_{L}\right.$ and $\left.C_{S}\right)$ for its own is meaningless and only their difference

$$
\Delta C \equiv C_{L}-C_{S}
$$

is meaningful, since the energy can always be shifted by a constant. Therefore one should expect to consider only their difference. However, since we have fixed the zero energy point there is still a possibility to look at each of the constants separately. Of course, that would depend on the way we have fixed the energy, but we will still find it usefull to compare the large distance constant of the different models and the lattice, as will be described later.

Next, we will check whether such constants do appear in the holographic models. In appendices $(\mathrm{D}),(\mathrm{F})$ and $(\mathrm{G})$ we calculate the values of $C_{L}$ and $C_{S}$ for the different cases, before fixing the zero energy point as in [23]'s notations. The results we get after fixing the zero energy point are listed in table (4). Of course, the results for $\Delta C$ do not depend on how we have fixed the zero point energy, therefore they are explained by the mentioned appendices.

From these results we can already conclude about the constant term in holography by itself. The values of $\Delta C$ in all the models under investigation are all non-zero, negative and very close to each other $\left(\Delta C \simeq-5 \frac{\sigma}{m_{g b}}\right)$, such that several remarks are to be made: 


\begin{tabular}{|c|c|c|c|c|}
\hline & $C_{L}\left[\frac{\sigma}{m_{g b}}\right.$ & $C_{S}\left[\frac{\sigma}{m_{g b}}\right\rfloor$ & $\Delta C\left[\frac{\sigma}{m_{g b}}\right]$ \\
\hline \hline$D_{3}$ brane & 0.59 & 7.39 & -6.8 \\
\hline$D_{4}$ brane & 1.35 & 5.3 & -4.48 \\
\hline non critical $A d S_{6}$ & -1.32 & 4.02 & -5.34 \\
\hline Klebanov Strassler & -1.44 & 2.93 & -4.37 \\
\hline
\end{tabular}

Table 4. The values of the constant terms for the different models in the fixed zero energy point convention.

- It seems that generically holography generates such a constant term. We can get some intuition about this feature of holography by thinking of how one would generate it in a flat space-time Nambu-Goto theory. While at short distances a tachyonic instability takes over such that there is no sense in talking about a constant term in this region, at long distances it does make sense, but as happens - it does not shows up (see eq. (1.1)). The only way of generating such a term at long distances is by adding a boundary term to the Nambu-Goto action. Therefore, since holoraphic models have a boundary by definition, we can expect them to have a constant term of the discussed type.

- We interpret $(-\Delta C)$ as the physical mass of the quarks which remains after subtracting their bare mass. Therefore it is crucial that the values of $\Delta C$ in all the models have the same sign.

- The fact that all the holographic models admits a similar $\Delta C$ value is very interesting.

Now, we will derive the values of the constant terms for the gauge theory, using the lattice results. By adjusting the theoretical prediction

$$
E=\sigma \ell-\frac{\pi}{12} \frac{1}{\ell}+C_{L}
$$

(where the first term is just the linear potential and the second one is the well-known Luscher term) to the measurements, we find $C_{L}$. Finding $C_{S}$ appears to be a subtle issue. In principle, one should compare the theoretical prediction of perturbation theory to the measurements, and derive $C_{S}$ in the same manner as for $C_{L}$. However, as was found in [29], the pertubative expression of the potential (up to three loops) fails in describing the potential even at the shortest lengths reached by the lattice simulations. Therefore, by adjusting this theoretical prediction to the shortest lattice measurement, we can only get a rough estimate for $C_{S}$. We are required to this point in the next paragraph, but first let us present the results, listed in table (5). We use theoretical predictions of 0,1 and 2 loop orders of perturbation theory, in order to see how much $C_{L}$ changes as we go to high order expressions. 


\begin{tabular}{|l|c|c|c|c|}
\hline & $C_{L} \frac{\sigma}{m_{g b}}$ & $C_{S} \frac{\sigma}{m_{g b}}$ & $\Delta C \frac{\sigma}{m_{g b}}$ \\
\hline \hline Lattice SU(3) 0-loop & -0.63 & -2.24 & 1.61 \\
\hline Lattice SU(3) 1-loop & -0.63 & 6.31 & -6.94 \\
\hline Lattice SU(3) 2-loop & -0.63 & 2.48 & -3.11 \\
\hline Lattice SU(2) 0-loop & -0.43 & -0.01 & -0.42 \\
\hline Lattice SU(2) 1-loop & -0.43 & 11.67 & -12.1 \\
\hline Lattice SU(2) 2-loop & -0.43 & 5.94 & -6.37 \\
\hline
\end{tabular}

Table 5. The values of the constant terms for $\mathrm{SU}(2)$ and $\mathrm{SU}(3)$ gauge theories. We used the data of $[22,23]$ to calculate them.

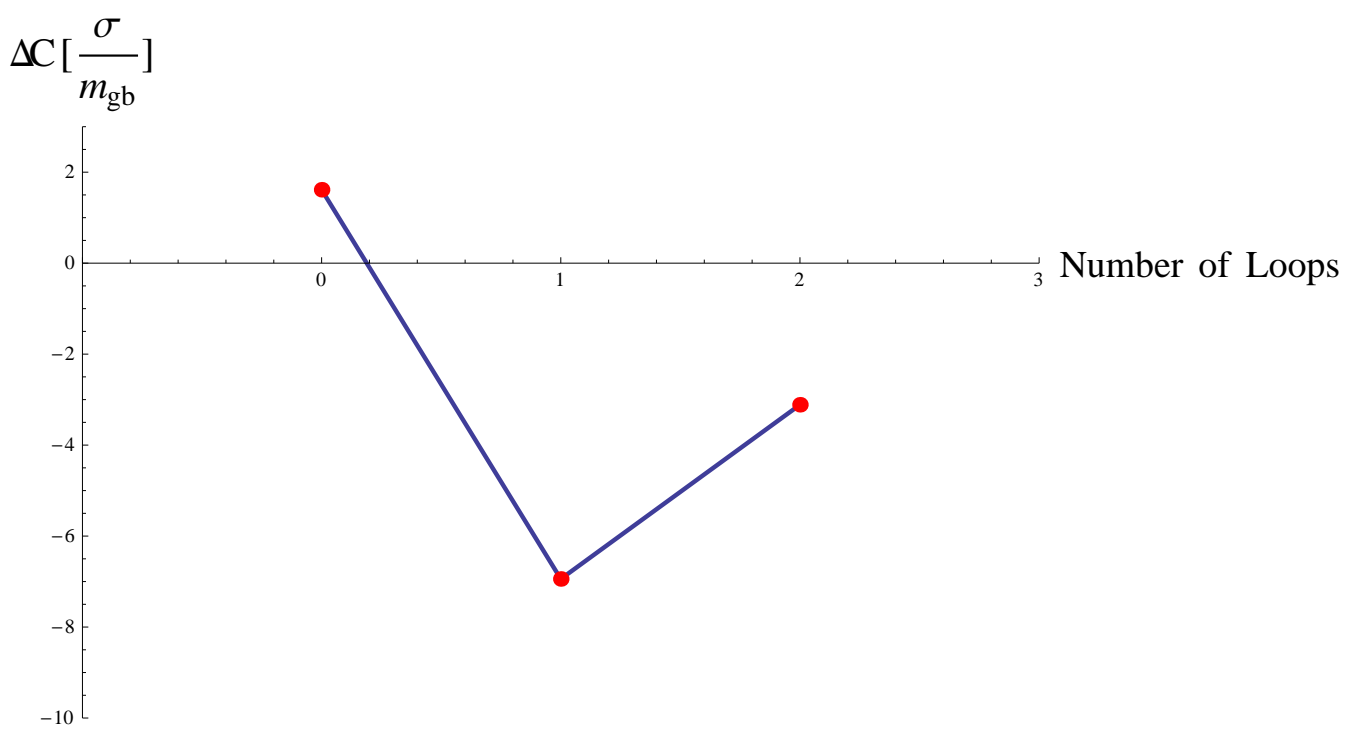

Figure 5. $\Delta C$ values as were calculated from comparison of the $\mathrm{SU}(3)$ lattice measurements to the theoretical predictions of 0,1 and 2 loops orders of perturbation theory.

As was discussed above, the process of finding the constant term at short distances (and therefore also $\Delta C$ ) out of the lattice measurements is a subtle issue. One way of resolving this issue is to consider only the constant at long distances, which is well defined since we have fixed the zero energy point, but also somewhat arbitrary defined, since this fixing is arbitrary. By examining the values of the constant terms at long distances one can notice that all the holographic models has $C_{L}$ 's which are of the same order as the one extracted out of the lattice data for the gauge theories. Moreover, our two favored candidates $\left(K S \& A d S_{6}\right)$ have negative $C_{L}$ as in the gauge theories (while the $\mathrm{D}$ branes have positive $C_{L}$ ), which may be regarded as another support in them. We emphasize that this conclusion should be taken with 
a grain of salt since it depends on how we have fixed the zero energy point, but it is still interesting. The other approach we take here is to look on the values of $\Delta C$ as were calculated from adjusting the theoretical predictions of 0,1 and 2 loops orders of perturbation theory to the lattice measurements and try to estimate to which value it would converge if we were able to use the theoretical expression of higher orders. From the results of the SU(3) measurements (plotted in figure (5)) the guess is that $\Delta C$ would converge to a value somewhat around $\Delta C=-5 \frac{\sigma}{m_{g b}}$. Considering this value as a basis for comparison, all the holographic models under investigation definitely have a very similar $\Delta C$ value.

\section{Concluding remarks \& future directions}

Following the statement made in the introduction, the goal of this paper is to search for a holographic background that admits a Wilson line which is as close as possible to the potential deduced from lattice calculations and which at the same time is in accordance with other requirements: Confinement at long distances, Coulombic potential at short distances (by which we mean $\frac{1}{\ell}$ behavior) ${ }^{10}$ and invariance under four dimensional Poincare symmetry.

We summarize by suggesting the Klebanov Strassler background as the holographic model which best fits the lattice measurements and in the same time is in accordance with the other requirements we have mentioned above ${ }^{11}$. Nevertheless, the non-critical $A d S_{6}$ also has an appreciable correspondence and it also admits the other requirements, so we cannot rule it out. However, from this point of view, the near extremal D branes can undoubtedly be excluded.

We turn now to final remarks and future directions. The first remark concerns the rank of the symmetry group, N. So far, the statement of holography (or the gauge/gravity duality) is made about dualities between string theories and field theories in the limit of large $\mathrm{N}$. On the other hand, we have used lattice simulations of $\mathrm{SU}(\mathrm{N})$ gauge theories with finite $\mathrm{N}(\mathrm{N}=2,3)$. Therefore one may argue that the comparison we have made is not valid. However, the claim is that the discussed Wilson line does not depends strongly on N. This claim is based on results from recent lattice simulations of Wilson lines [1-3] for several values of $\mathrm{N}$. It can also be seen in the lattice results we've been using (plotted in figure (3)), where there is no much difference between the $\mathrm{SU}(2)$ and $\mathrm{SU}(3)$ potentials. Assuming this claim to be correct, the large $\mathrm{N}$ Wilson line of the gauge theory should be similar to the ones we have used for the comparison.

The second remark concerns quantum corrections. As the analysis in the present work is only at the classical level, the arising question is how quantum corrections

\footnotetext{
${ }^{10}$ This requirement is motivated by the fact that the leading perturbative behavior of the QCD Wilson line is Coulombic.

${ }^{11}$ The KS potential at small distances is not exactly but rather approximately of the form $\sim \frac{1}{\ell}$.
} 
would affect the results. In order to understand this point, we first review the situation in the flat space-time N.G. case, there the potential at the classical level is just a linear piece

$$
E=\sigma \ell
$$

at all length scales. The full quantum expression (1.1) presents a dramatic change of this classical picture. While at long distances the quantum corrections are suppressed so the classical expression is a good approximation, at short length scales a tachyonic behavior takes over such that beyond some critical length (1.2) the full quantum expression (1.1) is not valid anymore. In the case of an asymptotically AdS curved background we expect the effect of the quantum corrections to be less dramatic, as we will explain below ${ }^{12}$. In the classical level, the potential is linear at long distances but it becomes Coulombic $\left(\sim \frac{1}{\ell}\right)$ at short distances. The quantum corrections at long distances were proven to be suppressed; in [30] ([31], [32]) the effective action of closed (open) confining strings was found to obey the Nambu-Goto form up to high orders, i.e. deviations from the N.G. energy levels are suppressed as some high power of $\frac{1}{\ell}$ (see also [33] where a discussion about quantum fluctuations of Wilson loop in string models is made). On dimensional grounds, the quantum corrections at short distances should have a Coulombic form (since the theory is asymptotically conformal) such that the potential would take the form

$$
\begin{aligned}
E & =-\frac{4 \pi^{2}}{\Gamma^{4}\left(\frac{1}{4}\right)} \frac{\sqrt{\lambda}}{\ell}\left[1+\frac{\tilde{\kappa}}{\sqrt{\lambda}}+\mathcal{O}\left(\frac{1}{\lambda}\right)\right] \\
\sqrt{\lambda} & \sim \frac{1}{\alpha^{\prime}}
\end{aligned}
$$

The one loop correction to the classical term was calculated in [34] and the value of $\tilde{\kappa}$ was evaluated numerically in $[35]^{13}$ and analytically in [36] to be

$$
\tilde{\kappa} \simeq-1.33460
$$

Therefore, the quantum corrections are suppressed in the limit where $\lambda \rightarrow \infty$ so at short enough distances the classical expression will be dominant and no tachyonic behavior would pop out. We stress that this is true only at large $\lambda$ (or equivalently weakly curved background) which is not necessarily the case in QCD. To emphasis the difference between the flat and curved space-time we remind that in the former

\footnotetext{
${ }^{12}$ However, there is an important difference between the cases. While the tachyonic behavior in the case of flat space-time NG is an indication for an instability of the theory, in the present construction of the curved space-time case it is an indication for an instability of the specific Wilson line we chose and not of the theory. The reason for this is that in the latter case we have constrained the string's endpoints to end on the boundary while in the former there are still dynamical open strings. Therefore, by referring here to instability of the curved background case we only mean instability of the Wilson line and not of the theory. We leave this issue for future work.

${ }^{13}$ We thank Nadav Drukker for referring us to this paper.
} 
case the expansion of the Wilson line is done in powers of $\frac{\alpha^{\prime}}{\ell^{2}}$ while in the latter the expansion is in powers of $\frac{1}{\sqrt{\lambda}}$ (or equivalently in powers of $\alpha^{\prime}$ ) due to the conformal symmetry. Hence, in the flat space-time scenario the quantum corrections at some given $\alpha^{\prime}$ would always become dominant at short enough distances and may present a tachyonic behavior, while in the asymptotically conformal curved space-time they are controllable. From this point of view we would prefer to consider the non-critical $A d S_{6}$ background rather than the $K S$ since the former is asymptotically AdS and the latter is not.

Concerning the issue of quantum corrections, some future directions are proposed:

- Following the statement made above, in asymptotically AdS backgrounds tachyonic behavior can arise only at intermediate distances, as opposed to the flat space-time case in which the tachyonic behavior is present below some critical length $\left(\ell<\ell_{c r}\right)$. It would be interesting to characterize this finite range of tachyonic behavior at intermediate distances and in particular try to formulate conditions under which it is going to zero, such that no tachyons are involved.

- Generalizing the quantum analysis to backgrounds which are not asymptotically AdS (like the $D_{p \neq 3}$ branes).

\section{Acknowledgments}

We are very grateful to Ofer Aharony for many useful discussions and for his critical comments on the manuscript. We would also like to thank Barak Bringoltz, Nadav Drukker and Herbert Neuberger for fruitful conversations. U.K. would like to thank Zohar Komargodski and Andrew Zayakin for helpful discussions during the Cargèse Summer School 2010 and to Dmitry Melnikov for assistance with the Klebanov-Strassler background. U.K. would like to dedicate a special thank to Shimon Yankielowicz for advising, teaching and supporting.

This work was supported in part by a centre of excellence supported by the Israel Science Foundation (grant number 1468/06), by a grant (DIP H52) of the German Israel Project Cooperation, by a BSF grant, by the European Network MRTN-CT2004-512194 and by European Union Excellence Grant MEXT-CT-2003-509661. 


\section{A The conditions on the background to satisfy a coulombic potential in the UV}

This derivation is being done using notations and symbols of [5]. First we expand the $f, g$ functions around $u=\infty$

$$
\begin{aligned}
f(u) & =a_{k} u^{k}+\mathcal{O}\left(u^{k-1}\right) \\
g(u) & =g(\infty)+b_{j} u^{-j}+\mathcal{O}\left(u^{-j+1}\right) \\
k, j & >0
\end{aligned}
$$

such that we can calculate the energy in the limit $u_{0} \gg u_{\Lambda}$ using these expansions. The length of the string is given by

$$
\begin{aligned}
\ell\left(u_{0}\right) & =2 f\left(u_{0}\right) \int_{u_{0}}^{\infty} d u \frac{g(u)}{f^{2}(u)} \frac{1}{\sqrt{1-\frac{f^{2}\left(u_{0}\right)}{f^{2}(u)}}} \\
& =2 a_{k} u_{0}^{k} \int_{u_{0}}^{\infty} d u \frac{g(\infty)+b_{j} u^{-j}}{a_{k}^{2} u^{2 k}} \frac{1}{\sqrt{1-\left(\frac{u_{0}}{u}\right)^{2 k}}} \\
& =2 a_{k} u_{0}^{k}\left[\frac{g(\infty)}{a_{k}^{2}} \int_{u_{0}}^{\infty} d u \frac{1}{u^{2 k}} \frac{1}{\sqrt{1-\left(\frac{u_{0}}{u}\right)^{2 k}}}+\frac{b_{j}}{a_{k}^{2}} \int_{u_{0}}^{\infty} d u \frac{1}{u^{2 k+j}} \frac{1}{\sqrt{1-\left(\frac{u_{0}}{u}\right)^{2 k}}}\right] \\
& =2 u_{0}^{k}\left[\frac{g(\infty)}{a_{k}} \frac{1}{\left(u_{0}\right)^{2 k-1}} \int_{1}^{\infty} d y \frac{1}{(y)^{2 k}} \frac{1}{\sqrt{1-y^{-2 k}}}+\frac{b_{j}}{a_{k}} \frac{1}{\left(u_{0}\right)^{2 k+j-1}} \int_{1}^{\infty} d \frac{1}{y^{2 k+j}} \frac{1}{\sqrt{1-y^{-2 k}}}\right] \\
& =\frac{2 g(\infty)}{a_{k}} \frac{1}{\left(u_{0}\right)^{k-1}} C_{2 k, 2 k}(\infty)+\frac{2 b_{j}}{a_{k}} \frac{1}{\left(u_{0}\right)^{k+j-1}} C_{2 k+j, 2 k}(\infty) \\
& =\left(\frac{2 g(\infty)}{a_{k}} C_{2 k, 2 k}(\infty)\right) \frac{1}{\left(u_{0}\right)^{k-1}}+\mathcal{O}\left(\frac{1}{\left(u_{0}\right)^{k+j-1}}\right)
\end{aligned}
$$

For the next steps we will need to use the invert function $u_{0}(\ell)$

$$
u_{0}(\ell)=\left(\frac{2 g(\infty)}{a_{k}} C_{2 k, 2 k}(\infty) \frac{1}{\ell}\right)^{\frac{1}{k-1}}
$$


The $\mathcal{K}$ function is given by

$$
\begin{aligned}
& \mathcal{K}\left(u_{0}\right)=\int_{u_{0}}^{\infty} d u g(u)\left[1-\sqrt{1-\frac{f^{2}\left(u_{0}\right)}{f^{2}(u)}}\right]+\int_{0}^{u_{0}} d u g(u) \\
& =\int_{u_{0}}^{\infty} d u\left(g(\infty)+b_{j} u^{-j}\right)\left[1-\sqrt{1-\left(\frac{u_{0}}{u}\right)^{2 k}}\right]+\int_{0}^{u_{0}} d u\left(g(\infty)+b_{j} u^{-j}\right) \\
& =\int_{1}^{\infty} d y u_{0}\left(g(\infty)+b_{j} y^{-j} u_{0}^{-j}\right)\left[1-\sqrt{1-y^{-2 k}}\right]+\int_{0}^{1} d u u_{0}\left(g(\infty)+b_{j} y^{-j} u_{0}^{-j}\right) \\
& =\frac{1}{2} u_{0} g(\infty) C_{2 k, 2 k}^{\prime}(\infty)+\frac{1}{2} u_{0}^{1-j} b_{j} C_{2 k+j, 2 k}^{\prime}(\infty) \\
& +u_{0} g(\infty)+\frac{b_{j}}{1-j} u_{0}^{1-j} \\
& =u_{0} g(\infty)\left[1+\frac{1}{2} C_{2 k, 2 k}^{\prime}(\infty)\right]+u_{0}^{1-j} b_{j}\left[\frac{1}{1-j}+\frac{1}{2} C_{2 k+j, 2 k}^{\prime}(\infty)\right] \\
& =u_{0} g(\infty)\left[1+\frac{1}{2} C_{2 k, 2 k}^{\prime}(\infty)\right]+\mathcal{O}\left(u_{0}^{1-j}\right)
\end{aligned}
$$

Using (A.3) we can express it as a function of $\ell$

$$
\mathcal{K}(\ell)=\left\{g(\infty)\left[1+\frac{1}{2} C_{2 k, 2 k}^{\prime}(\infty)\right]\left(\frac{2 g(\infty)}{a_{k}} C_{2 k, 2 k}(\infty)\right)^{\frac{1}{k-1}}\right\}\left(\frac{1}{\ell}\right)^{\frac{1}{k-1}}
$$

Finally, the energy of the string as a function of its length would be given by

$$
\begin{aligned}
E(\ell) & =f\left(u_{0}(\ell)\right) \cdot \ell-2 \mathcal{K}(\ell) \\
& =\left\{a_{k}\left(\frac{2 g(\infty)}{a_{k}} C_{2 k, 2 k}(\infty)\right)^{\frac{k}{k-1}}+g(\infty)\left[1+\frac{1}{2} C_{2 k, 2 k}^{\prime}(\infty)\right]\left(\frac{2 g(\infty)}{a_{k}} C_{2 k, 2 k}(\infty)\right)^{\frac{1}{k-1}}\right\}\left(\frac{1}{\ell}\right)^{\frac{1}{k-1}}
\end{aligned}
$$

The conclusion is that in order to have a coulombic behaviour at small distances we have to demand the following asymptotic form of $f$

$$
f\left(u \gg u_{\Lambda}\right) \sim u^{2}
$$

\section{B Conical singularity in near extremal $D_{p}$ brane}

We review the calculation of the periodicity of the compactified coordinate in the case of near extremal $D_{p}$ brane background. The part of the metric which includes the radial coordiante, $u$, and the compactified one, $x$, is given by

$$
d s^{2} \sim-\left(\frac{u}{R}\right)^{\frac{7-p}{2}}\left[1-\left(\frac{u_{\Lambda}}{u}\right)^{7-p}\right] d x^{2}+\frac{1}{\left(\frac{u}{R}\right)^{\frac{7-p}{2}}\left[1-\left(\frac{u_{\Lambda}}{u}\right)^{7-p}\right]} d u^{2}
$$


Following [37] let us define a new coordinate

$$
\frac{u}{u_{\Lambda}}=1+\left(\frac{\rho}{R}\right)^{2}
$$

such that the singularity at $u=u_{\Lambda}$ maps to $\rho=0$. Then for small $\rho$ the relevant $2 d$ part of the Euclidean metric $(\tau=i x)$ is given by

$$
d s^{2} \sim(7-p) u_{\Lambda}^{\frac{7-p}{2}} R^{-\frac{11-p}{2}} \rho^{2} d \tau^{2}+\frac{4}{7-p} u_{\Lambda}^{-\frac{3-p}{2}} R^{\frac{3-p}{2}} d \rho^{2}
$$

which can be written as [38]

$$
d s^{2} \sim \Omega\left[d \rho^{2}+c^{2} \rho^{2} d \tau^{2}\right]
$$

where

$$
\begin{aligned}
& \Omega=\frac{4}{7-p} u_{\Lambda}^{-\frac{3-p}{2}} R^{\frac{3-p}{2}} \\
& c^{2}=\frac{(7-p) u_{\Lambda}^{\frac{7-p}{2}} R^{-\frac{11-p}{2}}}{\Omega}=\left(\frac{7-p}{2}\right)^{2} u_{\Lambda}^{5-p} R^{-(7-p)}
\end{aligned}
$$

In order to avoid a conical singularity the coordinate $c \tau$ must be identified we a periodicity of $2 \pi$

$$
c \tau \rightarrow c \tau+2 \pi
$$

means the periodicity of $\tau$ is given by

$$
\beta=\frac{2 \pi}{c}=\frac{4 \pi}{7-p} u_{\Lambda}^{-\frac{5-p}{2}} R^{\frac{7-p}{2}}
$$

In a similar manner, the periodicity of the compactified coordinate of the non critical $A d S_{n+1}$ background was calculated in [39] to be

$$
\beta=\frac{4 \pi}{n} \frac{R^{2}}{u_{\Lambda}}
$$

\section{Analytical expressions for energy and length of the string in near extremal $D_{p}$ brane}

In the case of near extremal $D_{p}$ brane, the metric is of the form

$$
\begin{aligned}
& f^{2}(u)=\left(\frac{1}{2 \pi \alpha^{\prime}}\right)^{2}\left(\frac{u}{R_{A d S}}\right)^{7-p} \\
& g^{2}(u)=\left(\frac{1}{2 \pi \alpha^{\prime}}\right)^{2} \frac{1}{1-\left(\frac{u_{\Lambda}}{u}\right)^{7-p}}
\end{aligned}
$$


Inserting the above functions into the expression of the string's length (2.9) we have

$$
\begin{aligned}
\ell\left(u_{0}\right) & =2 \frac{1}{f\left(u_{0}\right)} \int_{u_{0}}^{u_{b}} d u \frac{f^{2}\left(u_{0}\right)}{f^{2}(u)} g(u) \frac{1}{\sqrt{1-\frac{f^{2}\left(u_{0}\right)}{f^{2}(u)}}} \\
& =2\left(\frac{R_{A d S}}{u_{\Lambda}}\right)^{\frac{7-p}{2}} \int_{u_{0}}^{\infty} d u\left(\frac{u_{0}}{u}\right)^{7-p} \frac{1}{\sqrt{1-\left(\frac{u_{\Lambda}}{u}\right)^{7-p}} \sqrt{1-\left(\frac{u_{0}}{u}\right)^{7-p}}}
\end{aligned}
$$

Define new variables

$$
\begin{aligned}
& y=\frac{u}{u_{0}} \\
& z=\left(\frac{u_{\Lambda}}{u_{0}}\right)^{7-p}
\end{aligned}
$$

we can write the length as a function of $z$

$$
\ell(z)=2 u_{\Lambda} \cdot\left(\frac{R_{A d S}}{u_{\Lambda}}\right)^{\frac{7-p}{2}} \cdot(z)^{\frac{5-p}{2(7-p)}} \cdot \int_{1}^{\infty} d y \frac{y^{-(7-p)}}{\sqrt{1-z y^{-(7-p)}} \sqrt{1-y^{-(7-p)}}}
$$

Notice that the length is measured in units of $u_{\Lambda} \cdot\left(\frac{R_{A d S}}{u_{\Lambda}}\right)^{\frac{7-p}{2}}$. Then we express it in terms of the universal system of units defined in (6) using the dictionary derived in section (7) (table (2)) to get

$$
\ell(z)=\frac{2 d_{p}}{m_{g b}}\left[(z)^{\frac{5-p}{2(7-p)}} \int_{1}^{\infty} d y \frac{y^{-(7-p)}}{\sqrt{1-z y^{-(7-p)}} \sqrt{1-y^{-(7-p)}}}\right]
$$

where $d_{p}=3.4,2.24$ for $p=3,4$, respectively. In a similar way we calculate the $\mathcal{K}$ function (2.13)

$$
\begin{aligned}
\mathcal{K}\left(u_{0}\right) & =\int_{u_{0}}^{u_{b}} d u g(u)\left(1-\sqrt{1-\frac{f^{2}\left(u_{0}\right)}{f^{2}(u)}}\right)+\int_{u_{\Lambda}}^{u_{0}} d u g(u) \\
& =\frac{1}{2 \pi \alpha^{\prime}}\left[\int_{u_{0}}^{\infty} d u \frac{1}{\sqrt{1-\left(\frac{u_{\Lambda}}{u}\right)^{7-p}}}\left(1-\sqrt{1-\left(\frac{u_{0}}{u}\right)^{7-p}}\right)+\int_{u_{\Lambda}}^{u_{0}} d u \frac{1}{\sqrt{1-\left(\frac{u_{\Lambda}}{u}\right)^{7-p}}}\right] \\
\text { C. } 7) & \\
\mathcal{K}(z) & =\frac{u_{\Lambda}}{\alpha^{\prime}} z^{-\frac{1}{7-p}} \frac{1}{2 \pi}\left[\int_{1}^{\infty} d y \frac{1-\sqrt{1-y^{-(7-p)}}}{\sqrt{1-z y^{-(7-p)}}}+\int_{z^{\frac{1}{7-p}}}^{1} d y \frac{1}{\sqrt{1-z y^{-(7-p)}}}\right] \quad \text { (C.8) }
\end{aligned}
$$

Notice that the energy, which has the same dimension as $\mathcal{K}$, is measured in units of $\frac{u_{\Lambda}}{\alpha^{\prime}}$. Expressing it in terms of the universal system of units we have

$$
\mathcal{K}(z)=\frac{\sigma}{m_{g b}} d_{p} z^{-\frac{1}{7-p}}\left[\int_{1}^{\infty} d y \frac{1-\sqrt{1-y^{-(7-p)}}}{\sqrt{1-z y^{-(7-p)}}}+\int_{z^{\frac{1}{7-p}}}^{1} d y \frac{1}{\sqrt{1-z y^{-(7-p)}}}\right]
$$


Finally, the energy is given by $(2.12)$

$$
\begin{aligned}
E\left(u_{0}\right)=f\left(u_{0}\right) \cdot \ell\left(u_{0}\right)-2 \mathcal{K}\left(u_{0}\right) & \text { (C.10) } \\
E(z)= & \frac{1}{2 \pi \alpha^{\prime}}\left(\frac{u_{\Lambda}}{R_{A d S}}\right)^{\frac{7-p}{2}} z^{-\frac{1}{2}} \cdot \ell(z)-2 \mathcal{K}(z) \\
= & \sigma z^{-\frac{1}{2}} \cdot \ell(z)-2 \mathcal{K}(z) \\
= & \frac{\sigma}{m_{g b}} 2 d_{p} z^{-\frac{1}{7-p}}\left[\int_{1}^{\infty} d y\left[\frac{1-\sqrt{1-y^{-(7-p)}}}{\sqrt{1-y^{-(7-p)}} \sqrt{1-z y^{-(7-p)}}}\right]-\int_{z^{\frac{1}{7-p}}}^{1} d y \frac{1}{\sqrt{1-z y^{-(7-p)}}}\right]
\end{aligned}
$$

\section{The values of the constant terms in near extremal $D_{p}$ brane}

In this appendix we will find the constant terms of the potential at short and long distances for the near extremal $D_{p}$ brane, using the expressions for the energy and length derived in appendix (C) using the mass subtraction regularization scheme. Pay attention that we are before fixing the zero energy point as in [23]'s notations. First, at short distances, i.e. the limit $z \rightarrow 0$, one finds

$$
E \rightarrow \frac{\sigma}{m_{g b}} 2 d_{p}\left[z^{-\frac{1}{7-p}} \int_{1}^{\infty} d y\left(\frac{1}{\sqrt{1-y^{-(7-p)}}}-1\right)-\frac{\sqrt{\pi} \Gamma\left[1-\frac{1}{7-p}\right]}{\Gamma\left[\frac{1}{2}-\frac{1}{7-p}\right]}+\ldots\right]
$$

where the three dots denote terms which vanish in this limit. The length is given by

$$
\ell \rightarrow \frac{1}{m_{g b}}(z)^{\frac{5-p}{2(7-p)}}\left[2 d_{p} \int_{1}^{\infty} d y \frac{y^{-(7-p)}}{\sqrt{1-y^{-(7-p)}}}\right]
$$

such that

$$
E=\left[\#_{p}\right] \cdot \sigma\left(m_{g b}\right)^{-\frac{7-p}{5-p}} \cdot \ell^{-\frac{2}{5-p}}-\frac{\sigma}{m_{g b}} \frac{2 d_{p} \sqrt{\pi} \Gamma\left[1-\frac{1}{7-p}\right]}{\Gamma\left[\frac{1}{2}-\frac{1}{7-p}\right]}
$$

In fact, we could alreay conclude this power law behavior by the analysis in (A), but the important point here is the value of the constant term

$$
C_{S}=-\frac{\sigma}{m_{g b}} \frac{2 d_{p} \sqrt{\pi} \Gamma\left[1-\frac{1}{7-p}\right]}{\Gamma\left[\frac{1}{2}-\frac{1}{7-p}\right]}
$$

Next we examine the behavior at long distances, i.e. $z \rightarrow 1$, there

$$
E=\sigma \cdot \ell-2 \mathcal{K}(z=1)
$$


such that the constant term is given by

$$
\begin{aligned}
C_{L} & =-2 \mathcal{K}(z=1) \\
& =-\frac{\sigma}{m_{g b}} 2 d_{p}\left[\int_{1}^{\infty} d y\left(\frac{1}{\sqrt{1-y^{-(7-p)}}}-1\right)\right]
\end{aligned}
$$

Finally, we conclude that the relative constant will be equal to

$$
\begin{aligned}
\Delta C & =C_{L}-C_{S} \\
& =-\frac{\sigma}{m_{g b}} 2 d_{p}\left[\frac{\sqrt{\pi} \Gamma\left[1-\frac{1}{7-p}\right]}{\Gamma\left[\frac{1}{2}-\frac{1}{7-p}\right]}+\int_{1}^{\infty} d y\left(\frac{1}{\sqrt{1-y^{-(7-p)}}}-1\right)\right]
\end{aligned}
$$

The expression in the square brackets appears to be equal to one such that

$$
\Delta C=-2 d_{p} \frac{\sigma}{m_{g b}}
$$

\begin{tabular}{|c|c|c|}
\hline$p$ & 3 & 4 \\
\hline \hline$\Delta C\left[\frac{\sigma}{m_{g b}}\right]$ & -6.8 & -4.48 \\
\hline
\end{tabular}

\section{E Analytical expressions for energy and length of the string in non-critical $A d S_{n+1}$}

Following the same steps as for the $D$ branes example $(\mathrm{C})$, the length of the string in the non-critical $A d S_{n+1}$ case is given by

$$
\ell\left(u_{0}\right)=2 \frac{R_{A d S}^{2}}{u_{0}^{2}} \int_{u_{0}}^{\infty} d u \frac{1}{\left(\frac{u}{u_{0}}\right)^{4} \sqrt{1-\left(\frac{u_{\Lambda}}{u}\right)^{n}} \sqrt{1-\left(\frac{u_{0}}{u}\right)^{4}}}
$$

Changing variables to $y \equiv \frac{u}{u_{0}}, z \equiv\left(\frac{u_{\Lambda}}{u_{0}}\right)^{n}$ we have

$$
=2 \frac{R_{A d S}^{2}}{u_{\Lambda}} z^{\frac{1}{n}} \int_{1}^{\infty} d y \frac{y^{-4}}{\sqrt{1-z y^{-n}} \sqrt{1-y^{-4}}}
$$

Notice that in the present case length is measured in units of $\frac{R_{A d S}^{2}}{u_{\Lambda}}$. Then expressing it in terms of the universal system of units defined in (6) using the dictionary derived in section (7) (table (2)) we result with

$$
\ell(z)=\frac{1}{m_{g b}}\left[5.04 z^{\frac{1}{n}} \int_{1}^{\infty} d y \frac{y^{-4}}{\sqrt{1-z y^{-n}} \sqrt{1-y^{-4}}}\right]
$$

In the same manner we derive the explicit expression for the $\mathcal{K}$ function 


$$
\begin{aligned}
\mathcal{K}\left(u_{0}\right) & =\frac{1}{2 \pi \alpha^{\prime}} \int_{u_{0}}^{\infty} d u \frac{1}{\sqrt{1-\left(\frac{u_{\Lambda}}{u}\right)^{n}}}\left(1-\sqrt{1-\left(\frac{u_{0}}{u}\right)^{4}}\right)+\frac{1}{2 \pi} \int_{u_{\Lambda}}^{u_{0}} d u \frac{1}{\sqrt{1-\left(\frac{u_{\Lambda}}{u}\right)^{n}}} \\
& =\frac{u_{\Lambda}}{\alpha^{\prime}} z^{-\frac{1}{n}} \frac{1}{2 \pi}\left[\int_{1}^{\infty} d y \frac{1}{\sqrt{1-z y^{-n}}}\left(1-\sqrt{1-y^{-4}}\right)+\frac{1}{2 \pi} \int_{z^{\frac{1}{n}}}^{1} d y \frac{1}{\sqrt{1-z y^{-n}}}\right]
\end{aligned}
$$

We see that as in the $D$ branes case, energy is measured in units of $\frac{u_{\Lambda}}{\alpha^{\prime}}$. In the universal system of units the $\mathcal{K}$ function will take the form

$$
\mathcal{K}(z)=\frac{\sigma}{m_{g b}}\left[2.52 z^{-\frac{1}{n}}\left(\int_{1}^{\infty} d y \frac{1}{\sqrt{1-z y^{-n}}}\left(1-\sqrt{1-y^{-4}}\right)+\int_{z^{\frac{1}{n}}}^{1} d y \frac{1}{\sqrt{1-z y^{-n}}}\right)\right]
$$

Finally the expression for the energy is given by

$$
E(z)=\sigma z^{-\frac{2}{n}} \cdot \ell(z)-2 \mathcal{K}(z)
$$

such that

$$
E(z)=\frac{\sigma}{m_{g b}} 5.04 z^{-\frac{1}{n}}\left[\int_{1}^{\infty} d y \frac{1-\sqrt{1-y^{-4}}}{\sqrt{1-z y^{-n}} \sqrt{1-y^{-4}}}-\int_{z^{\frac{1}{n}}}^{1} d y \frac{1}{\sqrt{1-z y^{-n}}}\right]
$$

\section{F The values of the constant terms in non-critical $A d S_{n+1}$}

Following again the same steps as for the $D$ brane cases in (D), we will find the constant terms of the energy in the cases of non-critical $A d S_{n+1}$. We will use the expressions for the energy and length derived in appendix (E). Pay attention that we are before fixing the zero energy point as in [23]'s notations. First, at short distances, i.e. the limit $z \rightarrow 0$, one finds

$$
E(z) \rightarrow \frac{\sigma}{m_{g b}} 5.04\left[z^{-\frac{1}{n}} \int_{1}^{\infty} d y\left(\frac{1}{\sqrt{1-y^{-4}}}-1\right)-\frac{\sqrt{\pi} \Gamma\left[1-\frac{1}{n}\right]}{\Gamma\left[\frac{1}{2}-\frac{1}{n}\right]}+\ldots\right]
$$

where the three dots denote terms which vanish in this limit. The length is given by

$$
\ell(z) \rightarrow \frac{1}{m_{g b}} 5.04 z^{\frac{1}{n}}\left[\int_{1}^{\infty} d y \frac{y^{-4}}{\sqrt{1-y^{-4}}}\right]
$$

such that

$E=\frac{\sigma}{m_{g b}^{2}}(5.04)^{2}\left[\int_{1}^{\infty} d y \frac{y^{-4}}{\sqrt{1-y^{-4}}}\right]\left[\int_{1}^{\infty} d y\left(\frac{1}{\sqrt{1-y^{-4}}}-1\right)-1\right] \frac{1}{\ell}-\frac{\sigma}{m_{g b}} \frac{5.04 \sqrt{\pi} \Gamma\left[1-\frac{1}{n}\right]}{\Gamma\left[\frac{1}{2}-\frac{1}{n}\right]}$ 


$$
E \cong-\left(0.36 \cdot(5.04)^{2} \frac{\sigma}{m_{g b}^{2}}\right) \frac{1}{\ell}-\frac{\sigma}{m_{g b}} \frac{5.04 \sqrt{\pi} \Gamma\left[1-\frac{1}{n}\right]}{\Gamma\left[\frac{1}{2}-\frac{1}{n}\right]}
$$

We find that the constant term at short distances is given by

$$
C_{S}=-\frac{\sigma}{m_{g b}} \frac{5.04 \sqrt{\pi} \Gamma\left[1-\frac{1}{n}\right]}{\Gamma\left[\frac{1}{2}-\frac{1}{n}\right]}
$$

In the opposite limit, $z \rightarrow 1$, corresponding to large distances, the energy takes the form

$$
E=\sigma \cdot \ell-2 \mathcal{K}(z=1)
$$

such that the constant term is equal to

$$
\begin{aligned}
C_{L} & =-2 \mathcal{K}(z=1) \\
& =-\frac{\sigma}{m_{g b}}\left[5.04\left(\int_{1}^{\infty} d y \frac{1-\sqrt{1-y^{-4}}}{\sqrt{1-y^{-n}}}\right)\right]
\end{aligned}
$$

Finally, we conclude that the relative constant will be equal to

$$
\begin{aligned}
\Delta C & =C_{L}-C_{S} \\
& =-\frac{\sigma}{m_{g b}} 5.04\left[\frac{\sqrt{\pi} \Gamma\left[1-\frac{1}{n}\right]}{\Gamma\left[\frac{1}{2}-\frac{1}{n}\right]}+\int_{1}^{\infty} d y \frac{1-\sqrt{1-y^{-4}}}{\sqrt{1-y^{-n}}}\right]
\end{aligned}
$$

and this can be evaluate numerically for different values of $n$ :

$$
\Delta C=-5.34
$$

\section{G The Wilson line of the Klebanov Strassler background}

The supergravity solution of the deformed conifold is of the following form [13, 27]

$$
d s^{2}=h^{-\frac{1}{2}}(\tau) d x_{0123}^{2}+h^{\frac{1}{2}}(\tau) d s_{6}^{2}
$$

where $d s_{6}^{2}$ is the metric of the deformed conifold

$d s_{6}^{2}=\frac{\epsilon^{4 / 3}}{2} K(\tau)\left[\frac{1}{3 K^{3}(\tau)}\left(d \tau^{2}+\left(g^{5}\right)^{2}\right)+\cosh ^{2}\left(\frac{\tau}{2}\right)\left[\left(g^{3}\right)^{2}+\left(g^{4}\right)^{2}\right]+\sinh ^{2}\left(\frac{\tau}{2}\right)\left[\left(g^{1}\right)^{2}+\left(g^{2}\right)^{2}\right]\right]$

$\epsilon$ is the energy scale and the functions $h(\tau)$ and $K(\tau)$ are given by

$$
\begin{gathered}
h(\tau)=\left(g_{s} M \alpha^{\prime}\right)^{2} 2^{2 / 3} \epsilon^{-8 / 3} I(\tau) \\
I(\tau) \equiv \int_{\tau}^{\infty} d x \frac{x \operatorname{coth} x-1}{\sinh ^{2} x}(\sinh 2 x-2 x)^{\frac{1}{3}}
\end{gathered}
$$




$$
K(\tau)=\frac{(\sinh (2 \tau)-2 \tau)^{\frac{1}{3}}}{2^{\frac{1}{3}} \sinh \tau}
$$

Therefore the $f$ and $g$ functions are

$$
\begin{aligned}
& f(\tau)=h^{-\frac{1}{2}}(\tau) \\
& g(\tau)=\frac{1}{\sqrt{6}} \epsilon^{2 / 3} K^{-1}(\tau)
\end{aligned}
$$

Near the boundary, i.e. at large $\tau$, the $h(\tau)$ and $K(\tau)$ function takes the form

$$
\begin{gathered}
h(\tau \gg 1)=\left(g_{s} M \alpha^{\prime}\right)^{2} 3 \cdot 2^{2 / 3} \epsilon^{-8 / 3} \tau e^{-\frac{4}{3} \tau} \\
K(\tau \gg 1)=2^{\frac{1}{3}} e^{-\frac{1}{3} \tau}
\end{gathered}
$$

Then the distance between the quarks is

$$
\begin{aligned}
\ell\left(\tau_{0}\right) & =\frac{2}{f\left(\tau_{0}\right)} \int_{\tau_{0}}^{\infty} d \tau g(\tau)\left[\frac{f\left(\tau_{0}\right)}{f(\tau)}\right]^{2} \frac{1}{\sqrt{1-\left[\frac{f\left(\tau_{0}\right)}{f(\tau)}\right]^{2}}} \\
& =\frac{2^{\frac{2}{3}} \epsilon^{2 / 3}}{\sqrt{6} f\left(\tau_{0}\right)} \int_{\tau_{0}}^{\infty} d \tau e^{\frac{1}{3} \tau}\left(\frac{\tau}{\tau_{0}} e^{-\frac{4}{3}\left(\tau-\tau_{0}\right)}\right) \frac{1}{\sqrt{1-\frac{\tau}{\tau_{0}} e^{-\frac{4}{3}\left(\tau-\tau_{0}\right)}}} \\
\left(t=\frac{\tau}{\tau_{0}}\right) & =\frac{2^{\frac{2}{3}} \epsilon^{2 / 3}}{\sqrt{6} f\left(\tau_{0}\right)} e^{+\frac{1}{3} \tau_{0}} \tau_{0} \int_{1}^{\infty} d t\left(t e^{-\tau_{0}(t-1)}\right) \frac{1}{\sqrt{1-t e^{-\frac{4}{3} \tau_{0}(t-1)}}}
\end{aligned}
$$

In the limit $\tau_{0} \rightarrow \infty$ the integral takes the value

$$
\tau_{0} \int_{1}^{\infty} d t\left(t e^{-\tau_{0}(t-1)}\right) \frac{1}{\sqrt{1-t e^{-\frac{4}{3} \tau_{0}(t-1)}}} \rightarrow 1.8
$$

such that

$$
\ell\left(\tau_{0}\right)=\frac{2^{\frac{2}{3}} \cdot 1.8 \cdot \epsilon^{2 / 3}}{\sqrt{6} f\left(\tau_{0}\right)} e^{+\frac{1}{3} \tau_{0}}=2^{1 / 2} \cdot 1.8 \cdot\left(g_{s} M \alpha^{\prime}\right) \epsilon^{-2 / 3} \tau_{0}^{1 / 2} e^{-\frac{1}{3} \tau_{0}}
$$

The energy of the pair is

$$
E\left(\tau_{0}\right)=f\left(\tau_{0}\right) \ell\left(\tau_{0}\right)-2 \mathcal{K}\left(\tau_{0}\right)
$$

where the $\mathcal{K}$ function is

$$
\begin{aligned}
\mathcal{K}\left(\tau_{0}\right) & =\int_{\tau_{0}}^{\infty} d \tau g(\tau)\left[1-\sqrt{1-\left[\frac{f\left(\tau_{0}\right)}{f(\tau)}\right]^{2}}\right]+\int_{0}^{\tau_{0}} d \tau g(\tau) \\
& =\frac{\epsilon^{2 / 3}}{2^{\frac{1}{3} \sqrt{6}}} \int_{\tau_{0}}^{\infty} d \tau e^{\frac{1}{3} \tau}\left[1-\sqrt{1-\frac{\tau}{\tau_{0}} e^{-\frac{4}{3}\left(\tau-\tau_{0}\right)}}\right]+\frac{\epsilon^{2 / 3}}{2^{\frac{1}{3}} \sqrt{6}} \int_{0}^{\tau_{0}} d \tau e^{\frac{1}{3} \tau} \\
\left(t=\frac{\tau}{\tau_{0}}\right) & =\frac{\epsilon^{2 / 3}}{2^{\frac{1}{3}} \sqrt{6}} \tau_{0} \int_{1}^{\infty} d t e^{\frac{1}{3} \tau_{0} t}\left[1-\sqrt{1-t e^{-\frac{4}{3} \tau_{0}(t-1)}}\right]+\frac{3 \epsilon^{2 / 3}}{2^{\frac{1}{3}} \sqrt{6}}\left(e^{\frac{1}{3} \tau_{0}}-1\right)
\end{aligned}
$$


Let us write this expression in the next form

$$
=\frac{\epsilon^{2 / 3}}{2^{\frac{1}{3}} \sqrt{6}} e^{\frac{1}{3} \tau_{0}} \tau_{0} \int_{1}^{\infty} d t e^{\frac{1}{3} \tau_{0}(t-1)}\left[1-\sqrt{1-t e^{-\frac{4}{3} \tau_{0}(t-1)}}\right]+\frac{3 \epsilon^{2 / 3}}{2^{\frac{1}{3}} \sqrt{6}}\left(e^{\frac{1}{3} \tau_{0}}-1\right)
$$

In the limit $\tau_{0} \rightarrow \infty$ the integral takes the value

$$
\tau_{0} \int_{1}^{\infty} d t e^{\frac{1}{3} \tau_{0}(t-1)}\left[1-\sqrt{1-t e^{-\frac{4}{3} \tau_{0}(t-1)}}\right] \rightarrow 0.6
$$

such that the Kappa function will take the form

$$
\mathcal{K}\left(\tau_{0}\right)=\frac{3.6 \epsilon^{2 / 3}}{2^{\frac{1}{3}} \sqrt{6}} e^{\frac{1}{3} \tau_{0}}-\frac{3 \epsilon^{2 / 3}}{2^{\frac{1}{3}} \sqrt{6}}
$$

Thus the energy in the region close to the boundary is given by

$$
\frac{E}{\epsilon^{2 / 3}}=-\frac{1.8 \cdot 2^{\frac{2}{3}}}{\sqrt{6}} e^{+\frac{1}{3} \tau_{0}}+\frac{3 \cdot 2^{\frac{2}{3}}}{\sqrt{6}}
$$

We would like to express the energy as a function of the distance between the quarks

$$
\ln \left[\left(g_{s} M \alpha^{\prime}\right)^{-1} \epsilon^{2 / 3} \ell\right]=-\frac{1}{3} \tau_{0}+\ln \left(\tau_{0}^{\frac{1}{2}}\right)+\ln \left(2^{1 / 2} \cdot 1.8\right)
$$

To first order (at large $\tau_{0}$ ) we have

$$
\ln \left[\left(g_{s} M \alpha^{\prime}\right)^{-1} \epsilon^{2 / 3} \ell\right] \cong-\frac{1}{3} \tau_{0}
$$

such that the leading behavior of the energy is indeed Coulombic

$$
\frac{E}{\epsilon^{2 / 3}} \cong-\frac{1.8 \cdot 2^{\frac{2}{3}}}{\sqrt{6}} \frac{\left(g_{s} M \alpha^{\prime}\right)}{\epsilon^{2 / 3} \ell}+\frac{3 \cdot 2^{\frac{2}{3}}}{\sqrt{6}}
$$

It is not a surprise since what we have done by taking the leading behavior (G.19) is exactly considering only the leading geometry, which is $A d S$, as discussed in section (5).

We now want to estimate the leading correction to the Coulombic potential. To do that we first find the leading correction to the dependence of the length on $\tau_{0}$ by plugging in the leading order (G.19) into the subleaing term of (G.18)

$$
\begin{aligned}
& \ln \left[\left(g_{s} M \alpha^{\prime}\right)^{-1} \epsilon^{2 / 3} \ell\right] \cong-\frac{1}{3} \tau_{0}+\ln \left(\sqrt{-\ln \left[\left(g_{s} M \alpha^{\prime}\right)^{-1} \epsilon^{2 / 3} \ell\right]}\right)+\ln \left(\sqrt{3} \cdot 2^{-\frac{2}{3}} \cdot 1.8\right) \\
& \frac{1}{3} \tau_{0} \cong-\ln \left[\left(g_{s} M \alpha^{\prime}\right)^{-1} \epsilon^{2 / 3} \ell\right]+\ln \left(\sqrt{-\ln \left[\left(g_{s} M \alpha^{\prime}\right)^{-1} \epsilon^{2 / 3} \ell\right]}\right)+\ln \left(\sqrt{3} \cdot 2^{-\frac{2}{3}} \cdot 1.8\right)
\end{aligned}
$$


Therefore the energy will take the form

$$
\frac{E}{\epsilon^{2 / 3}} \cong-\frac{1.8^{2}}{2} \frac{\sqrt{-\ln \left[\left(g_{s} M \alpha^{\prime}\right)^{-1} \epsilon^{2 / 3} \ell\right]}}{\left(g_{s} M \alpha^{\prime}\right)^{-1} \epsilon^{2 / 3} \ell}+\frac{3 \cdot 2^{\frac{2}{3}}}{\sqrt{6}}
$$

Until now we have worked in the natural system of units of the KS model, but in order to make a comparison we have to switch to the universal system, as follows from the dictionary derived in section (7) (table (2))

$$
\begin{aligned}
f(\tau) & =\left[2^{-\frac{1}{3}} I^{-\frac{1}{2}}(\tau)\right] \frac{\epsilon^{\frac{4}{3}}}{g_{s} M \alpha^{\prime}} \\
& =\left[\frac{1.51}{0.86} 2^{-\frac{1}{3}} I^{-\frac{1}{2}}(\tau)\right] \sigma \\
g(\tau) & =\left[\frac{1}{\sqrt{6}} K^{-1}(\tau)\right] \epsilon^{2 / 3} \\
& =\left[\frac{1.51}{\sqrt{6}} K^{-1}(\tau)\right] \frac{\sigma}{m_{g b}}
\end{aligned}
$$

The constant term of the potential in the small distances regime can be read off from (G.23)

$$
C_{S}=\frac{3 \cdot 2^{\frac{2}{3}}}{\sqrt{6}} \epsilon^{2 / 3} \cong 1.94 \epsilon^{2 / 3}=2.93 \frac{\sigma}{m_{g b}}
$$

On the other hand, the constant term at large distances can be calculated numerically to be

$$
C_{L}=-2 \mathcal{K}(\tau=0)=-1.44 \frac{\sigma}{m_{g b}}
$$

Therefore the relative constant is given by

$$
\Delta C=C_{L}-C_{S}=-4.37 \frac{\sigma}{m_{g b}}
$$

\section{References}

[1] A. Athenodorou, B. Bringoltz and M. Teper, "The closed string spectrum of SU(N) gauge theories in 2+1 dimensions," Phys. Lett. B 656, 132 (2007) [arXiv:0709.0693 [hep-lat]].

[2] A. Athenodorou, B. Bringoltz and M. Teper, "Closed flux tubes and their string description in $\mathrm{D}=3+1 \mathrm{SU}(\mathrm{N})$ gauge theories," arXiv:1007.4720 [hep-lat].

[3] B. B. Brandt, "Probing boundary-corrections to Nambu-Goto open string energy levels in 3d SU(2) gauge theory", arXiv:1010.3625 [hep-lat].

[4] J. F. Arvis, "The Exact Q Anti-Q Potential In Nambu String Theory", Phys. Lett. B 127, $106(1983)$. 
[5] Y. Kinar, E. Schreiber and J. Sonnenschein, "Q anti-Q potential from strings in curved spacetime: Classical results," Nucl. Phys. B 566, 103 (2000) [arXiv:hep-th/9811192].

[6] J. M. Maldacena, "Wilson loops in large N field theories," Phys. Rev. Lett. 80, 4859 (1998) [arXiv:hep-th/9803002].

[7] S. de Haro, S. N. Solodukhin and K. Skenderis, "Holographic reconstruction of spacetime and renormalization in the AdS/CFT correspondence," Commun. Math. Phys. 217, 595 (2001) [arXiv:hep-th/0002230].

[8] N. Drukker, D. J. Gross and H. Ooguri, "Wilson loops and minimal surfaces," Phys. Rev. D 60, 125006 (1999) [arXiv:hep-th/9904191].

[9] J. M. Maldacena, "Wilson loops in large N field theories," Phys. Rev. Lett. 80, 4859 (1998) [arXiv:hep-th/9803002].

[10] E. Witten, "Anti-de Sitter space, thermal phase transition, and confinement in gauge theories," Adv. Theor. Math. Phys. 2, 505 (1998) [arXiv:hep-th/9803131].

[11] S. Kuperstein and J. Sonnenschein, "Non-critical supergravity $(\mathrm{d}>1)$ and holography," JHEP 0407, 049 (2004) [arXiv:hep-th/0403254].

[12] S. Kuperstein and J. Sonnenschein, "Non-critical, near extremal AdS(6) background as a holographic laboratory of four dimensional YM theory", JHEP 0411, 026 (2004) [arXiv:hep-th/0411009].

[13] I. R. Klebanov and M. J. Strassler, "Supergravity and a confining gauge theory: Duality cascades and chiSB-resolution of naked singularities," JHEP 0008, 052 (2000) [arXiv:hep-th/0007191].

[14] M. Mia, K. Dasgupta, C. Gale and S. Jeon, Nucl. Phys. B 839, 187 (2010) [arXiv:0902.1540 [hep-th]].

M. Mia, K. Dasgupta, C. Gale and S. Jeon, Phys. Rev. D 82, 026004 (2010) [arXiv:1004.0387 [hep-th]].

M. Mia, K. Dasgupta, C. Gale and S. Jeon, Phys. Lett. B 694, 460 (2011) [arXiv:1006.0055 [hep-th]].

[15] Y. Hidaka and R. D. Pisarski, "Zero Point Energy of Renormalized Wilson Loops," Phys. Rev. D 80, 074504 (2009) [arXiv:0907.4609 [hep-ph]].

[16] J. Sonnenschein, "Stringy confining Wilson loops," arXiv:hep-th/0009146.

[17] U. Gursoy and E. Kiritsis, "Exploring improved holographic theories for QCD: Part I," JHEP 0802, 032 (2008) [arXiv:0707.1324 [hep-th]].

U. Gursoy, E. Kiritsis and F. Nitti, "Exploring improved holographic theories for QCD: Part II," JHEP 0802, 019 (2008) [arXiv:0707.1349 [hep-th]].

[18] D. f. Zeng, "Heavy quark potentials in some renormalization group revised AdS/QCD models," Phys. Rev. D 78, 126006 (2008) [arXiv:0805.2733 [hep-th]].

[19] H. Boschi-Filho, N. R. F. Braga and C. N. Ferreira, "Static strings in 
Randall-Sundrum scenarios and the quark anti-quark potential," Phys. Rev. D 73, 106006 (2006) [Erratum-ibid. D 74, 089903 (2006)] [arXiv:hep-th/0512295].

[20] A. Brandhuber, N. Itzhaki, J. Sonnenschein and S. Yankielowicz, "Wilson loops, confinement, and phase transitions in large N gauge theories from supergravity," JHEP 9806, 001 (1998) [arXiv:hep-th/9803263].

[21] A. Loewy and J. Sonnenschein, "On the holographic duals of N = 1 gauge dynamics," JHEP 0108, 007 (2001) [arXiv:hep-th/0103163].

[22] R. Sommer, "A New way to set the energy scale in lattice gauge theories and its applications to the static force and alpha-s in SU(2) Yang-Mills theory," Nucl. Phys. B 411, 839 (1994) [arXiv:hep-lat/9310022].

[23] S. Necco and R. Sommer, "The $\mathrm{N}(\mathrm{f})=0$ heavy quark potential from short to intermediate distances," Nucl. Phys. B 622, 328 (2002) [arXiv:hep-lat/0108008].

[24] C. Csaki, H. Ooguri, Y. Oz and J. Terning, "Glueball mass spectrum from supergravity," JHEP 9901, 017 (1999) [arXiv:hep-th/9806021].

[25] J. A. Minahan, "Glueball mass spectra and other issues for supergravity duals of QCD models," JHEP 9901, 020 (1999) [arXiv:hep-th/9811156].

[26] M. Berg, M. Haack and W. Mueck, "Glueballs vs. gluinoballs: Fluctuation spectra in non-AdS/non-CFT," Nucl. Phys. B 789, 1 (2008) [arXiv:hep-th/0612224].

[27] M. K. Benna, A. Dymarsky, I. R. Klebanov and A. Solovyov, "On Normal Modes of a Warped Throat," JHEP 0806, 070 (2008) [arXiv:0712.4404 [hep-th]].

[28] M. J. Teper, "SU(N) gauge theories in 2+1 dimensions," Phys. Rev. D 59, 014512 (1999) [arXiv:hep-lat/9804008].

[29] S. Necco and R. Sommer, "Testing perturbation theory on the $\mathrm{N}(\mathrm{f})=0$ static quark potential," Phys. Lett. B 523, 135 (2001) [arXiv:hep-ph/0109093].

[30] O. Aharony and E. Karzbrun, "On the effective action of confining strings," JHEP 0906, 012 (2009) [arXiv:0903.1927 [hep-th]].

[31] O. Aharony and M. Field, "On the effective theory of long open strings," arXiv:1008.2636 [hep-th].

[32] O. Aharony and N. Klinghoffer, "Corrections to Nambu-Goto energy levels from the effective string action," arXiv:1008.2648 [hep-th].

[33] Y. Kinar, E. Schreiber, J. Sonnenschein and N. Weiss, Nucl. Phys. B 583, 76 (2000) [arXiv:hep-th/9911123].

[34] N. Drukker, D. J. Gross and A. A. Tseytlin, "Green-Schwarz string in AdS(5) x S(5): Semiclassical partition function," JHEP 0004, 021 (2000) [arXiv:hep-th/0001204].

[35] S. x. Chu, D. Hou and H. c. Ren, "The Subleading Term of the Strong Coupling Expansion of the Heavy-Quark Potential in a $\mathcal{N}=4$ Super Yang-Mills Vacuum," JHEP 0908, 004 (2009) [arXiv:0905.1874 [hep-ph]]. 
[36] V. Forini, "Quark-antiquark potential in AdS at one loop," JHEP 1011, 079 (2010) [arXiv:1009.3939 [hep-th]].

[37] I. R. Klebanov, "TASI lectures: Introduction to the AdS/CFT correspondence," arXiv:hep-th/0009139.

[38] M. Alves, "The conical singularity method and the energy-momentum tensor near the black hole horizon using the Kruskal coordinates," Mod. Phys. Lett. A 22, 3057 (2007) [arXiv:0704.2013 [gr-qc]].

[39] G. T. Horowitz and R. C. Myers, "The AdS/CFT Correspondence and a New Positive Energy Conjecture for General Relativity," Phys. Rev. D 59, 026005 (1998) [arXiv:hep-th/9808079]. 